\title{
Structural and Thermal Investigations of $\mathrm{L}-\mathrm{CuC}_{4} \mathrm{H}_{4} \mathrm{O}_{6} \cdot 3 \mathrm{H}_{2} \mathrm{O}$ and DL- $\mathrm{CuC}_{4} \mathrm{H}_{4} \mathrm{O}_{6} \cdot 2 \mathrm{H}_{2} \mathrm{O}$ Single Crystals
}

\author{
Takanori Fukami ${ }^{1}$, Shuta Tahara ${ }^{1}$ \\ ${ }^{1}$ Department of Physics and Earth Sciences, Faculty of Science, University of the Ryukyus, Japan \\ Correspondence: Takanori Fukami, Department of Physics and Earth Sciences, Faculty of Science, University of the \\ Ryukyus, Okinawa 903-0213, Japan. E-mail: fukami@sci.u-ryukyu.ac.jp
}

Received: January 5, 2021 Accepted: March 31, $2021 \quad$ Online Published: April 10, 2021

doi:10.5539/ijc.v13n1p38

URL: https://doi.org/10.5539/ijc.v13n1p38

\begin{abstract}
Copper(II) L-tartrate trihydrate, $\mathrm{L}-\mathrm{CuC}_{4} \mathrm{H}_{4} \mathrm{O}_{6} \cdot 3 \mathrm{H}_{2} \mathrm{O}$, and copper(II) DL-tartrate dihydrate, $\mathrm{DL}-\mathrm{CuC}_{4} \mathrm{H}_{4} \mathrm{O}_{6} \cdot 2 \mathrm{H}_{2} \mathrm{O}$, crystals were grown at room temperature by the gel method using silica gels as the growth medium. Differential scanning calorimetry, thermogravimetric-differential thermal analysis, and X-ray diffraction measurements were performed on both crystals. The space group symmetries (monoclinic $P 2_{1}$ and $P 2_{1} / c$ ) and structural parameters of the crystals were determined at room temperature and at $114 \mathrm{~K}$. Both structures consisted of slightly distorted $\mathrm{CuO}_{6}$ octahedra, $\mathrm{C}_{4} \mathrm{H}_{4} \mathrm{O}_{6}$ and $\mathrm{H}_{2} \mathrm{O}$ molecules, $\mathrm{C}_{4} \mathrm{H}_{4} \mathrm{O}_{6}-\mathrm{Cu}-\mathrm{C}_{4} \mathrm{H}_{4} \mathrm{O}_{6}$ chains linked by $\mathrm{Cu}-\mathrm{O}$ bonds, and $\mathrm{O}-\mathrm{H}-\mathrm{O}$ hydrogen-bonding frameworks between adjacent molecules. Weight losses due to thermal decomposition of the crystals were found to occur in the temperature range of 300-1250 K. We inferred that the weight losses were caused by the evaporation of bound water molecules and the evolution of $\mathrm{H}_{2} \mathrm{CO}, \mathrm{CO}$, and $\mathrm{O}_{2}$ gases from $\mathrm{C}_{4} \mathrm{H}_{4} \mathrm{O}_{6}$ molecules, and that the residual reddish-brown substance left in the vessels after decomposition was copper(I) oxide $\left(\mathrm{Cu}_{2} \mathrm{O}\right)$.
\end{abstract}

Keywords: $\mathrm{L}-\mathrm{CuC}_{4} \mathrm{H}_{4} \mathrm{O}_{6} \cdot 3 \mathrm{H}_{2} \mathrm{O}$, DL- $\mathrm{CuC}_{4} \mathrm{H}_{4} \mathrm{O}_{6} \cdot 2 \mathrm{H}_{2} \mathrm{O}$, crystal structure, thermal decomposition, X-ray diffraction, TG-DTA

\section{Introduction}

Many tartrate compounds are formed by the reaction of tartaric acid with compounds containing positive ions (i.e., two monovalent cations or one divalent cation) (Desai \& Patel, 1988; Fukami, Hiyajyo, Tahara, \& Yasuda, 2017; Fukami \& Tahara, 2018; Fukami \& Tahara, 2020; Labutina, Marychev, Portnov, Somov, \& Chuprunov, 2011). Tartaric acid (chemical formula: $\mathrm{C}_{4} \mathrm{H}_{6} \mathrm{O}_{6}$; systematic name: 2,3-dihydroxybutanedioic acid) has two chiral carbon atoms in its structure, which provides the possibility for four possible different forms of chiral, racemic, and achiral isomers: L(+)-tartaric, D(-)-tartaric, racemic (DL-) tartaric, and meso-tartaric acid (Bootsma \& Schoone, 1967; Fukami, Tahara, Yasuda, \& Nakasone, 2016; Song, Teng, Dong, Ma, \& Sun, 2006). Some of these compounds are of interest because of their physical properties, particularly their excellent dielectric, ferroelectric, piezoelectric, and nonlinear optical properties (Abdel-Kader et al., 1991; Firdous, Quasim, Ahmad, \& Kotru, 2010; Torres et al., 2002). Moreover, they were formerly used in numerous industrial applications, for example, as transducers and in linear and non-linear mechanical devices.

Several experimental studies have been conducted on copper(II) tartrate crystals. Jethva et al. (Jethva, Dabhi, \& Joshi, 2016) reported the powder X-ray diffraction (XRD) patterns, Fourier-transform infrared spectroscopy and electron paramagnetic resonance spectroscopy analyses, magnetic properties, and thermal characteristics of copper L-tartrate and copper D-tartrate crystals grown by the gel method. Thermogravimetric analysis indicated that the L- and D-tartrate crystals contain three or half water molecules. Additionally, the crystals were reduced to copper oxide $(\mathrm{CuO})$ by thermal decomposition. Moreover, the powder XRD patterns indicated that the structures of the L- and D-tartrate crystals containing half water molecules are orthorhombic with lattice constants of $a=8.3700(7), b=12.8490(10)$, and $c=$ $8.7586(6) \AA$, and of $a=8.3650(14), b=12.8350(13)$, and $c=8.7580(9) \AA$, respectively. Labutina et al. (Labutina, Marychev, Portnov, Somov, \& Chuprunov, 2011) have grown many tartrate single crystals by the gel method, and reported their crystal system and lattice constants. The crystal structure of $\mathrm{CuC}_{4} \mathrm{H}_{4} \mathrm{O}_{6} \cdot 3 \mathrm{H}_{2} \mathrm{O}$, containing three water molecules, was found to be monoclinic with space group $P 2_{1}$ and lattice constants of $a=8.3632(8), b=8.7543(7), c=$ 12.1270(9) $\AA$, and $\beta=104.632(8)^{\circ}$. In other studies, the structures of copper tartrate crystals were reported as orthorhombic with cell parameters of $a=16.26, b=8.698$, and $c=7.469 \AA$, and as triclinic, space group $P \overline{1}$ (Binitha \& 
Pradyumnan, 2013; Bridle \& Lomer, 1965).

As mentioned above, it is expected that a copper(II) DL-tartrate compound can be synthesized using DL-tartaric acid and $\mathrm{Cu}(\mathrm{II})^{2+}$ ions as the divalent cations. The crystal structure of the resulting compound, $\mathrm{CuC}_{4} \mathrm{H}_{4} \mathrm{O}_{6} \cdot 3 \mathrm{H}_{2} \mathrm{O}$, containing three water molecules has not been determined yet, except for its crystal system and lattice constants. In this paper, we describe the synthesis of copper(II) L-tartrate trihydrate $\left(\mathrm{L}-\mathrm{CuC}_{4} \mathrm{H}_{4} \mathrm{O}_{6} \cdot 3 \mathrm{H}_{2} \mathrm{O}\right)$ and copper(II) DL-tartrate dihydrate $\left(\mathrm{DL}-\mathrm{CuC}_{4} \mathrm{H}_{4} \mathrm{O}_{6} \cdot 2 \mathrm{H}_{2} \mathrm{O}\right.$ ) crystals by the gel method, and determine their crystal structures using single-crystal X-ray diffraction. Moreover, the thermal properties of these crystals are studied by differential scanning calorimetry (DSC) and thermogravimetric-differential thermal analysis (TG-DTA).

\section{Experimental}

\subsection{Crystal Growth}

The $\mathrm{L}-\mathrm{CuC}_{4} \mathrm{H}_{4} \mathrm{O}_{6} \cdot 3 \mathrm{H}_{2} \mathrm{O}$ and DL-CuC $\mathrm{H}_{4} \mathrm{O}_{6} \cdot 2 \mathrm{H}_{2} \mathrm{O}$ crystals were grown in silica gel medium at room temperature using single test tube diffusion method. The gels for the growth were prepared in test tubes (with length of $200 \mathrm{~mm}$, and diameter of $30 \mathrm{~mm}$ ) using aqueous solutions of $\mathrm{Na}_{2} \mathrm{SiO}_{3}(20 \mathrm{ml}$ of $1 \mathrm{M}), \mathrm{L}_{-} \mathrm{C}_{4} \mathrm{H}_{6} \mathrm{O}_{6}$ (or DL- $\left.\mathrm{C}_{4} \mathrm{H}_{6} \mathrm{O}_{6}\right)(25 \mathrm{ml}$ of $1 \mathrm{M}$ ), and $\mathrm{CH}_{3} \mathrm{COOH}$ ( $25 \mathrm{ml}$ of $1 \mathrm{M}$ for the L-compound and $25 \mathrm{ml}$ of $1.5 \mathrm{M}$ for the DL-compound). The gels were aged for seven days, and solutions of $\mathrm{CuSO}_{4} \cdot 5 \mathrm{H}_{2} \mathrm{O}(30 \mathrm{ml}$ of $0.5 \mathrm{M})$ were then gently poured on top of the gels. The $\mathrm{L}-\mathrm{CuC}_{4} \mathrm{H}_{4} \mathrm{O}_{6} \cdot 3 \mathrm{H}_{2} \mathrm{O}$ and DL- $\mathrm{CuC}_{4} \mathrm{H}_{4} \mathrm{O}_{6} \cdot 2 \mathrm{H}_{2} \mathrm{O}$ crystals were harvested after about two and four months, respectively. Figure 1 shows the photograph of (a) $\mathrm{L}-\mathrm{CuC}_{4} \mathrm{H}_{4} \mathrm{O}_{6} \cdot 3 \mathrm{H}_{2} \mathrm{O}$ and (b) DL-CuC ${ }_{4} \mathrm{H}_{4} \mathrm{O}_{6} \cdot 2 \mathrm{H}_{2} \mathrm{O}$ single crystals grown in the gel medium. The $\mathrm{L}-\mathrm{CuC}_{4} \mathrm{H}_{4} \mathrm{O}_{6} \cdot 3 \mathrm{H}_{2} \mathrm{O}$ crystals, which are prism in shape and blue in color, are very similar to those reported in the previous paper (Jethva, Dabhi, \& Joshi, 2016).

(a)

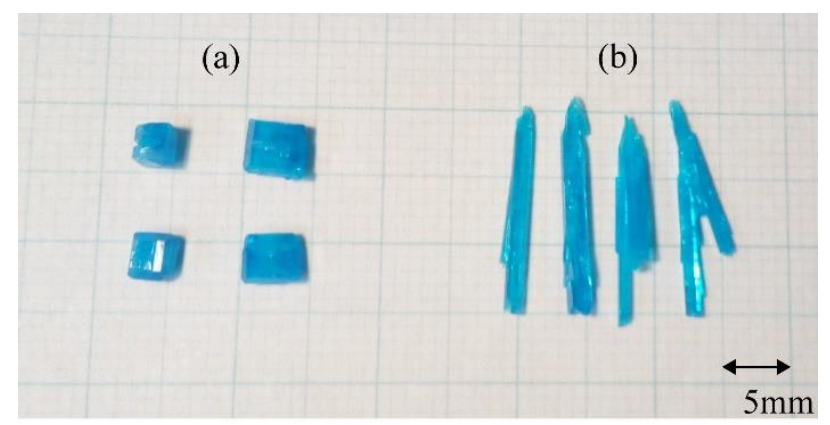

Figure 1. Photograph of (a) $\mathrm{L}-\mathrm{CuC}_{4} \mathrm{H}_{4} \mathrm{O}_{6} \cdot 3 \mathrm{H}_{2} \mathrm{O}$ and (b) DL- $\mathrm{CuC}_{4} \mathrm{H}_{4} \mathrm{O}_{6} \cdot 2 \mathrm{H}_{2} \mathrm{O}$ single crystals grown in the gel medium

\subsection{Structure Determination}

The X-ray diffraction measurements were performed using a Rigaku Saturn CCD X-ray diffractometer with graphite-monochromated Mo $K_{\alpha}$ radiation $(\lambda=0.71073 \AA)$. The diffraction data for the $\mathrm{L}_{-} \mathrm{CuC}_{4} \mathrm{H}_{4} \mathrm{O}_{6} \cdot 3 \mathrm{H}_{2} \mathrm{O}$ and DL- $\mathrm{CuC}_{4} \mathrm{H}_{4} \mathrm{O}_{6} \cdot 2 \mathrm{H}_{2} \mathrm{O}$ crystals were collected at 299 and $114 \mathrm{~K}$, respectively, using an $\omega$ scan mode with a crystal-to-detector distance of $40 \mathrm{~mm}$, and processed using the CrystalClear software package. The intensity data were corrected for Lorentz polarization and absorption effects. The crystal structures were solved by direct methods using the SIR2014 program and refined on $F^{2}$ by full-matrix least-squares methods using the SHELXL-2017 program in the WinGX package (Burla et al., 2015; Farrugia, 2012; Sheldrick, 2015).

\subsection{Thermal Measurements}

DSC and TG-DTA measurements were carried out in the temperature ranges of 100-310 and 300-1250 K, respectively, using DSC7020 and TG-DTA7300 systems from Seiko Instruments Inc. Aluminium (for DSC) and platinum (for TG-DTA) open pans were used as measuring vessels and reference pans. Fine powder samples of $\mathrm{L}_{-} \mathrm{CuC}_{4} \mathrm{H}_{4} \mathrm{O}_{6} \cdot 3 \mathrm{H}_{2} \mathrm{O}$ and $\mathrm{DL}-\mathrm{CuC}_{4} \mathrm{H}_{4} \mathrm{O}_{6} \cdot 2 \mathrm{H}_{2} \mathrm{O}$ for the thermal measurements were obtained by grinding several pieces of single crystals. The sample amount varied between 5.29 and $7.81 \mathrm{mg}$, and the heating rates were $10 \mathrm{~K} \mathrm{~min}^{-1}$ under the flow of nitrogen gas

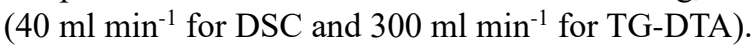

\section{Results and Discussion}

\subsection{Crystal Structures}

The crystal structures of $\mathrm{L}-\mathrm{CuC}_{4} \mathrm{H}_{4} \mathrm{O}_{6} \cdot 3 \mathrm{H}_{2} \mathrm{O}$ and DL-CuC $\mathrm{CH}_{4} \mathrm{O}_{6} \cdot 2 \mathrm{H}_{2} \mathrm{O}$ were determined at room temperature and at 114 $\mathrm{K}$, respectively. The lattice parameters calculated from all the observed X-ray reflections showed that both crystals belong to a monoclinic system. The systematic extinctions of the reflections from $\mathrm{L}-\mathrm{CuC}_{4} \mathrm{H}_{4} \mathrm{O}_{6} \cdot 3 \mathrm{H}_{2} \mathrm{O}$ revealed that the 
space group is $P 2_{1}$ or $P 2_{1} / m$, and the examination of the intensities of symmetry-equivalent reflections indicated that the crystal belongs to a non-centric space group. Therefore, the structure of $\mathrm{L}-\mathrm{CuC}_{4} \mathrm{H}_{4} \mathrm{O}_{6} \cdot 3 \mathrm{H}_{2} \mathrm{O}$ was determined to be monoclinic with space group $P 2_{1}$. On the other hand, the systematic extinctions from DL-CuC $\mathrm{H}_{4} \mathrm{H}_{6} \cdot 2 \mathrm{H}_{2} \mathrm{O}$ revealed that the space group is $P 2_{1} / c$. Consequently, the structure of DL-CuC $\mathrm{CH}_{4} \mathrm{O}_{6} \cdot 2 \mathrm{H}_{2} \mathrm{O}$ was found to be monoclinic with space group $P 2_{1} / c$. Some isotropic thermal parameters for hydrogen atoms belonging to water molecules in $\mathrm{L}-\mathrm{CuC}_{4} \mathrm{H}_{4} \mathrm{O}_{6} \cdot 3 \mathrm{H}_{2} \mathrm{O}$ were fixed during the structural refinement because the parameters did not converge to reasonable values. Final $R$-factors of $2.56 \%$ and $4.61 \%$ for the $\mathrm{L}-\mathrm{CuC}_{4} \mathrm{H}_{4} \mathrm{O}_{6} \cdot 3 \mathrm{H}_{2} \mathrm{O}$ and DL- $\mathrm{CuC}_{4} \mathrm{H}_{4} \mathrm{O}_{6} \cdot 2 \mathrm{H}_{2} \mathrm{O}$ crystals, respectively, were calculated for 7938 and 5283 unique observed reflections. The largest residual electron density peak and hole in the final difference Fourier map of DL- $\mathrm{CuC}_{4} \mathrm{H}_{4} \mathrm{O}_{6} \cdot 2 \mathrm{H}_{2} \mathrm{O}$ were 5.813 and -1.310 e $\AA^{-3}$, respectively, as shown in Table 1. From the following experimental results, we believe that the presence of the large values is mainly caused by radiation damage to the sample during the $\mathrm{X}$-ray data collection at $114 \mathrm{~K}$.

Firstly, X-ray measurements on the DL- $\mathrm{CuC}_{4} \mathrm{H}_{4} \mathrm{O}_{6} \cdot 2 \mathrm{H}_{2} \mathrm{O}$ crystal were performed at room temperature, similarly to those for $\mathrm{L}-\mathrm{CuC}_{4} \mathrm{H}_{4} \mathrm{O}_{6} \cdot 3 \mathrm{H}_{2} \mathrm{O}$. However, good quality X-ray intensity data for structural refinement could not be collected. Because Bragg peak splitting and several weak diffraction rings were observed in CCD diffraction images on the PC monitor, during the measurement of setting parameters for the intensity measurements or during data collection shortly after the determination of the setting parameters (monoclinic with lattice constants $a=8.778(14), b=11.141(19), c=$ 16.141(27) $\AA$, and $\beta=92.70(3)^{\circ}$ ). These results indicated that the sample crystals of DL- $\mathrm{CuC}_{4} \mathrm{H}_{4} \mathrm{O}_{6} \cdot 2 \mathrm{H}_{2} \mathrm{O}$ are easily damaged by X-ray irradiation at room temperature, and other crystalline and amorphous phases are formed in the samples.

Table 1. Crystal data, intensity data collections, and structure refinements for (a) $\mathrm{L}-\mathrm{CuC}_{4} \mathrm{H}_{4} \mathrm{O}_{6} \cdot 3 \mathrm{H}_{2} \mathrm{O}$ and (b) DL- $\mathrm{CuC}_{4} \mathrm{H}_{4} \mathrm{O}_{6} \cdot 2 \mathrm{H}_{2} \mathrm{O}$

\begin{tabular}{lll}
\hline & (a) & $(\mathrm{b})$ \\
\hline Compound, $M_{\mathrm{r}}$ & $\mathrm{CuO}_{9} \mathrm{C}_{4} \mathrm{H}_{10}, 265.66$ & $\mathrm{CuO}_{8} \mathrm{C}_{4} \mathrm{H}_{8}, 247.65$ \\
Measurement temperature & $299 \mathrm{~K}$ & $114 \mathrm{~K}$ \\
Crystal system, space group & Monoclinic, $P 2_{1}$ & Monoclinic, $P 2_{1} / c$ \\
Lattice constants & $a=8.3708(2) \AA$ & $a=8.7157(2) \AA$ \\
& $b=8.7602(1) \AA$ & $b=11.0467(2) \AA$ \\
& $c=12.1373(3) \AA$ & $c=16.1842(5) \AA$ \\
$V, Z$ & $\beta=104.538(1)^{\circ}$ & $\beta=92.669(1)^{\circ}$ \\
$D$ (cal.) & $861.53(3) \AA^{3}, 4$ & $1556.52(7) \AA^{3}, 8$ \\
$\mu\left(\right.$ Mo $\left.K_{\alpha}\right)$ & $2.048 \mathrm{Mg} \mathrm{m}^{-3}$ & $2.114 \mathrm{Mg} \mathrm{m}^{-3}$ \\
$F(000)$ & $2.565 \mathrm{~mm}^{-1}$ & $2.822 \mathrm{~mm}^{-1}$ \\
Crystal size & 540 & 1000 \\
$\theta$ range for data collection & $0.18 \times 0.18 \times 0.20 \mathrm{~mm}^{3}$ & $0.20 \times 0.20 \times 0.25 \mathrm{~mm}^{3}$ \\
Index ranges & $1.73-38.08^{\circ}$ & $2.34-32.00^{\circ}$ \\
Reflections collected, unique & $-14 \leq h \leq 14,-15 \leq k \leq 15,-21 \leq l \leq 20$ & $-12 \leq h \leq 12,-16 \leq k \leq 16,-24 \leq l \leq 24$ \\
Completeness to $\theta_{\max }$ & $24852,8784[R(\mathrm{int})=0.0215]$ & $33419,5390[R(\mathrm{int})=0.0382]$ \\
Absorption correction type & $94.9 \%$ & $99.9 \%$ \\
Transmission factor $T_{\min }-T_{\max }$ & $0.5530-0.6687$ & $\mathrm{Numerical}$ \\
Date, parameter & $7938[\mathrm{I}>2 \sigma(I)], 324$ & $0.5950-0.7026$ \\
Final $R$ indices & $R_{1}=0.0256, w R_{2}=0.0630$ & $5283[\mathrm{I}>2 \sigma(I)], 300$ \\
$R$ indices (all data) & $R_{1}=0.0299, w R_{2}=0.0656$ & $R_{1}=0.0461, w R_{2}=0.1139$ \\
Weighting scheme & $w=1 /\left[\sigma^{2}\left(F_{\mathrm{o}}^{2}\right)+(0.0402 P)^{2}\right]$ & $R_{1}=0.0472, w R_{2}=0.1148$ \\
& $P=\left(F_{\mathrm{o}}{ }^{2}+2 F_{\mathrm{c}}{ }^{2}\right) / 3$ & $P=1 /\left[\sigma^{2}\left(F_{\mathrm{o}}{ }^{2}\right)+(0.0482 P)^{2}+7.448 P\right]$ \\
Flack parameter & $-0.020(3)$ & \\
Goodness-of-fit on $F^{2}$ & 0.971 & \\
Extinction coefficient & $0.022(1)$ & 1.073 \\
Largest diff. peak and hole & $0.586 /-0.603 \mathrm{e} \AA^{-3}$ & $0.0004(3)$ \\
\hline
\end{tabular}


(a) $\mathrm{L}_{-} \mathrm{CuC}_{4} \mathrm{H}_{4} \mathrm{O}_{6} \cdot 3 \mathrm{H}_{2} \mathrm{O}$

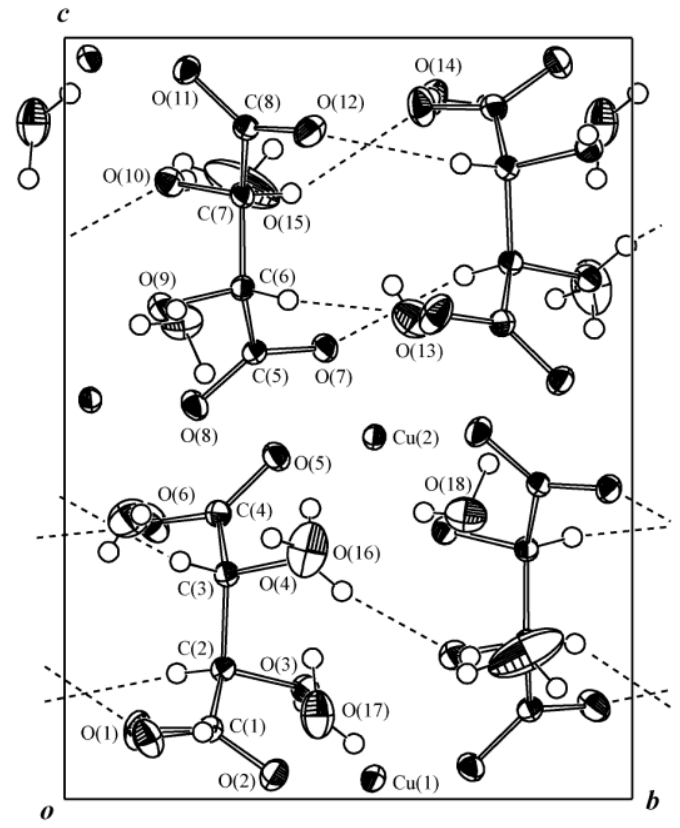

(b) $\mathrm{DL}_{-} \mathrm{CuC}_{4} \mathrm{H}_{4} \mathrm{O}_{6} \cdot 2 \mathrm{H}_{2} \mathrm{O}$

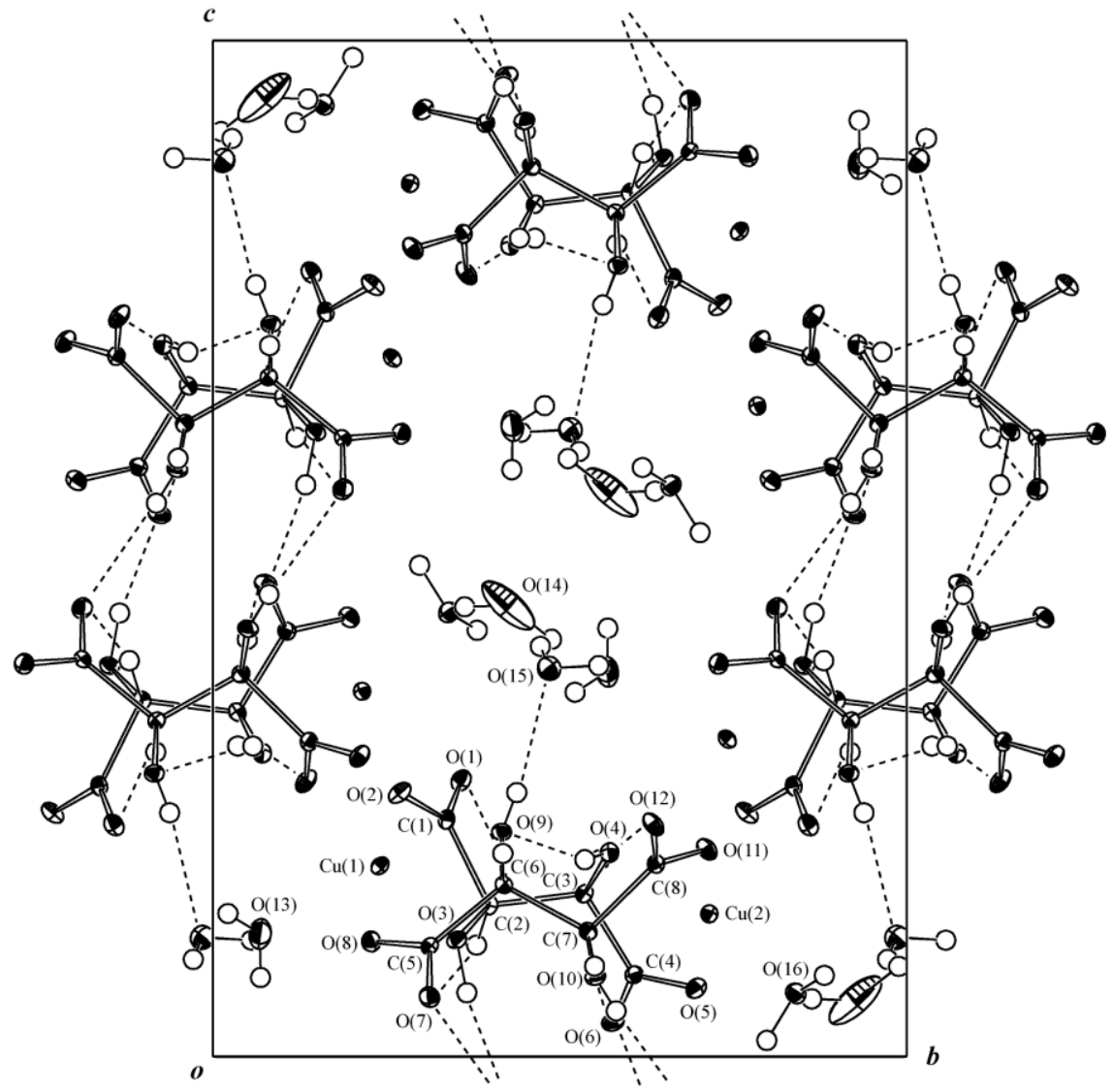

Figure 2. ORTEP projections of the crystal structures along the $a$-axis for (a) $\mathrm{L}-\mathrm{CuC}_{4} \mathrm{H}_{4} \mathrm{O}_{6} \cdot 3 \mathrm{H}_{2} \mathrm{O}$ (at room temperature) and (b) $\mathrm{DL}-\mathrm{CuC}_{4} \mathrm{H}_{4} \mathrm{O}_{6} \cdot 2 \mathrm{H}_{2} \mathrm{O}$ (at $114 \mathrm{~K}$ ), with $50 \%$ probability-displacement thermal ellipsoids

The solid and dashed short lines indicate $\mathrm{O}-\mathrm{H} \cdots \mathrm{O}$ hydrogen bonds of $\mathrm{C}_{4} \mathrm{H}_{4} \mathrm{O}_{6}$ molecules, as listed in Table 4 . 
Table 2. Atomic coordinates and thermal parameters $\left(\times 10^{4} \AA^{2}\right)$ for (a) $\mathrm{L}_{-} \mathrm{CuC}_{4} \mathrm{H}_{4} \mathrm{O}_{6} \cdot 3 \mathrm{H}_{2} \mathrm{O}$ (at room temperature) and (b) DL- $\mathrm{CuC}_{4} \mathrm{H}_{4} \mathrm{O}_{6} \cdot 2 \mathrm{H}_{2} \mathrm{O}$ (at $114 \mathrm{~K}$ ), with standard deviations in parentheses. The anisotropic thermal parameters are defined as $\exp \left[-2 \pi^{2}\left(U_{11} a^{* 2} h^{2}+U_{22} b^{* 2} k^{2}+U_{33} c^{* 2} l^{2}+2 U_{23} b^{*} c^{*} k l+2 U_{13} a^{*} c^{*} h l+2 U_{12} a^{*} b^{*} h k\right)\right]$. The isotropic thermal parameters $\left(\AA^{2}\right)$ for $\mathrm{H}$ atoms are listed under $U_{11}$

\begin{tabular}{|c|c|c|c|c|c|c|c|c|c|}
\hline \multicolumn{10}{|c|}{ (a) $\mathrm{L}-\mathrm{CuC}_{4} \mathrm{H}_{4} \mathrm{O}_{6} \cdot 3 \mathrm{H}_{2} \mathrm{O}$} \\
\hline Atom & $X$ & $y$ & $z$ & $U_{11}$ & $U_{22}$ & $U_{33}$ & $U_{23}$ & $U_{13}$ & $U_{12}$ \\
\hline $\mathrm{Cu}(1)$ & $0.19221(3)$ & $0.54412(3)$ & $0.02696(2)$ & $216(1)$ & $146.7(8)$ & 178.1(9) & 20.1(9) & $53.7(7)$ & $23(1)$ \\
\hline $\mathrm{Cu}(2)$ & $0.33158(2)$ & $0.54587(2)$ & $0.47550(2)$ & $181.8(9)$ & $126.1(8)$ & $156.1(8)$ & $4.6(9)$ & $-1.2(6)$ & $-6(1)$ \\
\hline $\mathrm{C}(1)$ & $0.3112(2)$ & $0.2561(2)$ & $0.0915(2)$ & $204(8)$ & $170(7)$ & $174(7)$ & $11(5)$ & $46(6)$ & $30(6)$ \\
\hline $\mathrm{C}(2)$ & $0.1973(2)$ & $0.2797(2)$ & $0.1708(1)$ & $166(7)$ & $149(6)$ & $146(6)$ & $8(5)$ & $28(6)$ & $-1(5)$ \\
\hline$C(3)$ & $0.2985(2)$ & $0.2857(2)$ & $0.2952(1)$ & $165(7)$ & $146(6)$ & $153(6)$ & $0(5)$ & $23(6)$ & $1(5)$ \\
\hline $\mathrm{C}(4)$ & $0.1834(2)$ & $0.2709(2)$ & $0.3746(2)$ & $216(8)$ & $166(7)$ & $176(7)$ & $-1(6)$ & $49(6)$ & $-33(6)$ \\
\hline$C(5)$ & $0.5900(2)$ & $0.3350(2)$ & $0.5869(2)$ & 195(7) & $142(6)$ & $144(6)$ & $11(5)$ & $0(6)$ & $14(5)$ \\
\hline$C(6)$ & $0.7589(2)$ & $0.3135(2)$ & $0.6716(2)$ & $154(7)$ & $137(6)$ & $151(6)$ & $4(5)$ & $-5(6)$ & $-4(5)$ \\
\hline$C(7)$ & $0.7393(2)$ & $0.3134(2)$ & $0.7936(2)$ & $179(8)$ & $139(6)$ & $156(7)$ & $-17(5)$ & $-6(6)$ & $4(6)$ \\
\hline $\mathrm{C}(8)$ & $0.9066(2)$ & $0.3197(2)$ & $0.8822(2)$ & 192(8) & $160(7)$ & $151(6)$ & $-10(5)$ & $-7(6)$ & $-23(6)$ \\
\hline $\mathrm{O}(1)$ & $0.3760(2)$ & $0.1291(2)$ & $0.0902(2)$ & $369(9)$ & $185(6)$ & $383(9)$ & $38(6)$ & $188(7)$ & $92(6)$ \\
\hline $\mathrm{O}(2)$ & $0.3355(2)$ & $0.3694(2)$ & $0.0320(1)$ & $287(7)$ & 193(6) & $242(6)$ & $46(5)$ & $129(6)$ & $52(5)$ \\
\hline $\mathrm{O}(3)$ & $0.1151(2)$ & $0.4236(2)$ & $0.1451(1)$ & $162(5)$ & $185(5)$ & $182(5)$ & $4(4)$ & $18(4)$ & $36(4)$ \\
\hline $\mathrm{O}(4)$ & $0.3951(2)$ & $0.4217(2)$ & $0.3156(1)$ & $167(6)$ & $184(5)$ & $183(5)$ & $23(5)$ & $13(4)$ & $-33(4)$ \\
\hline $\mathrm{O}(5)$ & $0.1841(2)$ & $0.3731(2)$ & $0.4499(1)$ & $250(7)$ & $182(6)$ & $201(6)$ & $-37(5)$ & $80(5)$ & $-48(5)$ \\
\hline $\mathrm{O}(6)$ & $0.0972(2)$ & $0.1544(2)$ & $0.3629(2)$ & $410(9)$ & $277(7)$ & $389(9)$ & $-117(7)$ & $215(8)$ & $-179(7)$ \\
\hline $\mathrm{O}(7)$ & $0.5170(2)$ & $0.4585(2)$ & $0.5925(1)$ & $253(7)$ & $159(5)$ & $195(6)$ & $-15(4)$ & $-33(5)$ & $59(5)$ \\
\hline $\mathrm{O}(8)$ & $0.5367(2)$ & $0.2293(2)$ & $0.5175(1)$ & $246(7)$ & $172(6)$ & $217(6)$ & $-44(5)$ & $-69(5)$ & $43(5)$ \\
\hline $\mathrm{O}(9)$ & $0.8246(2)$ & $0.1708(2)$ & $0.6467(1)$ & $153(5)$ & $172(5)$ & $202(6)$ & $-12(4)$ & $23(5)$ & $16(4)$ \\
\hline $\mathrm{O}(10)$ & $0.6439(2)$ & $0.1853(2)$ & $0.8106(1)$ & $175(6)$ & $207(6)$ & $211(6)$ & $-20(5)$ & $38(5)$ & $-35(5)$ \\
\hline $\mathrm{O}(11)$ & $0.9435(2)$ & $0.2157(2)$ & $0.9581(1)$ & $223(6)$ & $175(5)$ & 192(6) & $30(5)$ & $-30(5)$ & $-30(5)$ \\
\hline $\mathrm{O}(12)$ & $0.9942(2)$ & $0.4324(2)$ & $0.8775(1)$ & $321(8)$ & $250(7)$ & $245(7)$ & $63(6)$ & $-61(6)$ & $-136(6)$ \\
\hline $\mathrm{O}(13)$ & $0.2199(3)$ & $0.6139(2)$ & $0.6309(2)$ & $373(10)$ & $429(11)$ & $359(10)$ & $-61(8)$ & $144(8)$ & $-11(8)$ \\
\hline $\mathrm{O}(14)$ & $0.2998(2)$ & $0.6476(2)$ & $0.9202(2)$ & $299(8)$ & $231(7)$ & $358(9)$ & $82(6)$ & $142(7)$ & $14(6)$ \\
\hline $\mathrm{O}(15)$ & $0.3545(4)$ & $0.3127(5)$ & $0.8079(3)$ & $490(14)$ & $1413(30)$ & $595(16)$ & $-539(19)$ & $256(13)$ & $-64(18)$ \\
\hline $\mathrm{O}(16)$ & $0.7234(3)$ & $0.4280(3)$ & $0.3277(3)$ & $362(11)$ & $399(11)$ & $754(17)$ & $83(11)$ & 231(11) & $34(9)$ \\
\hline $\mathrm{O}(17)$ & $0.7841(3)$ & $0.4458(2)$ & $0.1111(2)$ & $335(9)$ & $265(8)$ & $570(13)$ & $-11(8)$ & 166(9) & $44(7)$ \\
\hline $\mathrm{O}(18)$ & $0.8798(2)$ & $0.7069(3)$ & $0.3735(2)$ & $276(8)$ & $428(10)$ & $297(8)$ & $-22(7)$ & $118(7)$ & $16(7)$ \\
\hline $\mathrm{H}(1)$ & $0.127(3)$ & $0.198(3)$ & $0.165(2)$ & $0.013(6)$ & & & & & \\
\hline $\mathrm{H}(2)$ & $0.371(3)$ & $0.204(3)$ & $0.312(2)$ & $0.012(6)$ & & & & & \\
\hline $\mathrm{H}(3)$ & $0.826(4)$ & $0.394(4)$ & $0.656(2)$ & $0.027(7)$ & & & & & \\
\hline $\mathrm{H}(4)$ & $0.682(3)$ & $0.399(3)$ & $0.796(2)$ & $0.016(6)$ & & & & & \\
\hline $\mathrm{H}(5)$ & $0.027(4)$ & $0.422(4)$ & $0.127(3)$ & 0.05 & & & & & \\
\hline $\mathrm{H}(6)$ & $0.362(4)$ & $0.489(4)$ & $0.273(3)$ & 0.05 & & & & & \\
\hline $\mathrm{H}(7)$ & $0.932(5)$ & $0.197(4)$ & $0.643(3)$ & $0.04(1)$ & & & & & \\
\hline $\mathrm{H}(8)$ & $0.561(5)$ & $0.214(5)$ & $0.813(3)$ & $0.05(1)$ & & & & & \\
\hline $\mathrm{H}(9)$ & $0.104(5)$ & $0.635(5)$ & $0.626(4)$ & $0.06(1)$ & & & & & \\
\hline $\mathrm{H}(10)$ & $0.244(5)$ & $0.578(5)$ & $0.675(4)$ & 0.07 & & & & & \\
\hline $\mathrm{H}(11)$ & $0.273(3)$ & $0.744(3)$ & $0.912(2)$ & $0.021(6)$ & & & & & \\
\hline $\mathrm{H}(12)$ & $0.386(5)$ & $0.644(5)$ & $0.921(3)$ & $0.05(1)$ & & & & & \\
\hline $\mathrm{H}(13)$ & $0.347(6)$ & $0.209(6)$ & $0.835(4)$ & 0.07 & & & & & \\
\hline $\mathrm{H}(14)$ & $0.326(6)$ & $0.367(5)$ & $0.854(4)$ & 0.07 & & & & & \\
\hline $\mathrm{H}(15)$ & $0.689(6)$ & $0.433(6)$ & $0.382(4)$ & 0.07 & & & & & \\
\hline $\mathrm{H}(16)$ & $0.788(5)$ & $0.365(5)$ & $0.343(4)$ & 0.07 & & & & & \\
\hline $\mathrm{H}(17)$ & $0.760(5)$ & $0.437(5)$ & $0.185(4)$ & 0.07 & & & & & \\
\hline $\mathrm{H}(18)$ & $0.739(5)$ & $0.510(5)$ & $0.073(4)$ & 0.07 & & & & & \\
\hline $\mathrm{H}(19)$ & $0.853(5)$ & $0.633(5)$ & $0.377(4)$ & $0.06(1)$ & & & & & \\
\hline
\end{tabular}




\begin{tabular}{|c|c|c|c|c|c|c|c|c|c|}
\hline \multirow{2}{*}{\multicolumn{10}{|c|}{$\begin{array}{lll}\mathrm{H}(20) & 0.830(5) & 0.747(4) \\
\text { (b) } \mathrm{DL}-\mathrm{CuC}_{4} \mathrm{H}_{4} \mathrm{O}_{6} \cdot 2 \mathrm{H}_{2} \mathrm{O}\end{array}$}} \\
\hline & & & & & & & & & \\
\hline $\mathrm{Cu}(1)$ & $0.74655(4)$ & $0.24113(3)$ & $0.18755(2)$ & $70(1)$ & $82(2)$ & $82(2)$ & $20(1)$ & $5(1)$ & $8(1)$ \\
\hline $\mathrm{Cu}(2)$ & $0.73206(4)$ & $0.71545(3)$ & $0.14037(2)$ & $65(1)$ & $74(1)$ & $82(2)$ & $1(1)$ & $-2(1)$ & $-6(1)$ \\
\hline $\mathrm{C}(1)$ & $0.4703(3)$ & $0.3368(2)$ & $0.2335(2)$ & $88(10)$ & $70(10)$ & $98(10)$ & $7(8)$ & $8(8)$ & $-12(8)$ \\
\hline $\mathrm{C}(2)$ & $0.4861(3)$ & $0.3981(2)$ & $0.1492(2)$ & $58(9)$ & $80(10)$ & $94(10)$ & $4(8)$ & $5(8)$ & $0(8)$ \\
\hline$C(3)$ & $0.4947(3)$ & $0.5354(2)$ & $0.1607(2)$ & $59(9)$ & $77(10)$ & $87(10)$ & $12(8)$ & $-2(8)$ & $-4(8)$ \\
\hline$C(4)$ & $0.4661(3)$ & $0.6069(2)$ & $0.0808(2)$ & $79(10)$ & $82(10)$ & $81(10)$ & $4(8)$ & $10(8)$ & $19(8)$ \\
\hline$C(5)$ & $0.0139(3)$ & $0.3127(2)$ & $0.1089(2)$ & 111(10) & $71(10)$ & $73(10)$ & $10(8)$ & $3(8)$ & $8(8)$ \\
\hline$C(6)$ & $0.0149(3)$ & $0.4193(2)$ & $0.1690(2)$ & $83(10)$ & $76(10)$ & $76(10)$ & $-5(8)$ & $7(8)$ & $3(8)$ \\
\hline$C(7)$ & $0.0135(3)$ & $0.5406(2)$ & $0.1233(2)$ & $74(10)$ & $82(10)$ & $81(10)$ & $-6(8)$ & $-1(8)$ & $-6(8)$ \\
\hline $\mathrm{C}(8)$ & $0.0342(3)$ & $0.6386(2)$ & $0.1899(2)$ & $76(10)$ & $75(10)$ & $90(10)$ & $-1(8)$ & $22(8)$ & $-17(8)$ \\
\hline $\mathrm{O}(1)$ & $0.3517(2)$ & $0.3579(2)$ & $0.2719(1)$ & $93(8)$ & $104(8)$ & $119(8)$ & $24(7)$ & $33(6)$ & $13(6)$ \\
\hline $\mathrm{O}(2)$ & $0.5793(2)$ & $0.2695(2)$ & $0.2598(1)$ & $92(8)$ & $130(9)$ & $113(9)$ & $50(7)$ & $24(7)$ & $25(7)$ \\
\hline $\mathrm{O}(3)$ & $0.6220(2)$ & $0.3516(2)$ & $0.1151(1)$ & $100(8)$ & $100(8)$ & $79(8)$ & $15(6)$ & $20(6)$ & $29(6)$ \\
\hline $\mathrm{O}(4)$ & $0.6383(2)$ & $0.5715(2)$ & $0.2010(1)$ & $72(8)$ & $88(8)$ & $99(8)$ & $1(6)$ & $-15(6)$ & $5(6)$ \\
\hline $\mathrm{O}(5)$ & $0.5534(2)$ & $0.6965(2)$ & $0.0674(1)$ & $92(8)$ & $106(8)$ & $105(8)$ & $22(7)$ & $-14(6)$ & $-24(6)$ \\
\hline $\mathrm{O}(6)$ & $0.3550(2)$ & $0.5766(2)$ & $0.0342(1)$ & $92(8)$ & 133(9) & $87(8)$ & $18(7)$ & $-15(6)$ & $-15(7)$ \\
\hline $\mathrm{O}(7)$ & $0.1186(2)$ & $0.3123(2)$ & $0.0586(1)$ & $139(9)$ & $106(8)$ & $110(8)$ & $-9(7)$ & $52(7)$ & $-8(7)$ \\
\hline $\mathrm{O}(8)$ & $-0.0848(2)$ & $0.2284(2)$ & $0.1133(1)$ & $104(8)$ & $92(8)$ & $111(8)$ & $-2(6)$ & $25(7)$ & $-6(6)$ \\
\hline $\mathrm{O}(9)$ & $-0.1131(2)$ & $0.4162(2)$ & $0.2212(1)$ & $89(8)$ & $101(8)$ & $76(8)$ & $-1(6)$ & $16(6)$ & $7(6)$ \\
\hline $\mathrm{O}(10)$ & $-0.1298(2)$ & $0.5509(2)$ & $0.0787(1)$ & $115(8)$ & $109(8)$ & $76(8)$ & $8(7)$ & $-19(6)$ & $-8(7)$ \\
\hline $\mathrm{O}(11)$ & $-0.0724(2)$ & $0.7118(2)$ & $0.2042(1)$ & $79(8)$ & $105(8)$ & $121(8)$ & $-36(7)$ & $-9(6)$ & $9(6)$ \\
\hline $\mathrm{O}(12)$ & $0.1628(2)$ & $0.6346(2)$ & $0.2287(1)$ & $75(8)$ & $115(8)$ & 133(9) & $-53(7)$ & $-20(6)$ & $5(6)$ \\
\hline $\mathrm{O}(13)$ & $0.6294(3)$ & $0.0684(2)$ & $0.1209(2)$ & $358(14)$ & $126(10)$ & $243(13)$ & $16(9)$ & $-89(11)$ & $-12(9)$ \\
\hline $\mathrm{O}(14)$ & $0.6588(4)$ & $0.4257(5)$ & $0.4443(3)$ & $257(15)$ & $735(28)$ & $628(24)$ & $-486(23)$ & $114(15)$ & $-143(17)$ \\
\hline $\mathrm{O}(15)$ & $0.9539(3)$ & $0.4848(2)$ & $0.3824(1)$ & $203(11)$ & $142(10)$ & $151(10)$ & $-7(8)$ & $31(8)$ & $24(8)$ \\
\hline $\mathrm{O}(16)$ & $0.8094(2)$ & $0.8399(2)$ & $0.0624(1)$ & $126(9)$ & $105(8)$ & $110(8)$ & $-11(7)$ & $29(7)$ & $-31(7)$ \\
\hline $\mathrm{H}(1)$ & $0.405(5)$ & $0.380(4)$ & $0.110(3)$ & $0.02(1)$ & & & & & \\
\hline $\mathrm{H}(2)$ & $0.421(5)$ & $0.558(4)$ & $0.194(3)$ & $0.02(1)$ & & & & & \\
\hline $\mathrm{H}(3)$ & $0.108(5)$ & $0.418(4)$ & $0.200(3)$ & $0.02(1)$ & & & & & \\
\hline $\mathrm{H}(4)$ & $0.098(6)$ & $0.550(5)$ & $0.089(3)$ & $0.02(1)$ & & & & & \\
\hline $\mathrm{H}(5)$ & $0.638(7)$ & $0.366(6)$ & $0.063(4)$ & $0.06(2)$ & & & & & \\
\hline $\mathrm{H}(6)$ & $0.686(8)$ & $0.536(6)$ & $0.194(4)$ & $0.06(2)$ & & & & & \\
\hline $\mathrm{H}(7)$ & $-0.097(7)$ & $0.438(6)$ & $0.260(4)$ & $0.05(2)$ & & & & & \\
\hline $\mathrm{H}(8)$ & $-0.119(6)$ & $0.581(5)$ & $0.045(3)$ & $0.02(1)$ & & & & & \\
\hline $\mathrm{H}(9)$ & $0.661(8)$ & $0.022(6)$ & $0.141(4)$ & $0.05(2)$ & & & & & \\
\hline $\mathrm{H}(10)$ & $0.653(8)$ & $0.069(6)$ & $0.078(4)$ & $0.05(2)$ & & & & & \\
\hline $\mathrm{H}(11)$ & $0.621(9)$ & $0.363(8)$ & $0.443(5)$ & $0.09(2)$ & & & & & \\
\hline $\mathrm{H}(12)$ & $0.567(10)$ & $0.488(8)$ & $0.411(5)$ & $0.16(3)$ & & & & & \\
\hline $\mathrm{H}(13)$ & $0.993(6)$ & $0.556(5)$ & $0.385(3)$ & $0.02(1)$ & & & & & \\
\hline $\mathrm{H}(14)$ & $0.867(8)$ & $0.472(6)$ & $0.404(4)$ & $0.06(2)$ & & & & & \\
\hline $\mathrm{H}(15)$ & $0.890(6)$ & $0.881(5)$ & $0.080(3)$ & $0.03(1)$ & & & & & \\
\hline $\mathrm{H}(16)$ & $0.838(6)$ & $0.798(5)$ & $0.016(3)$ & $0.03(1)$ & & & & & \\
\hline
\end{tabular}


Table 3. Selected interatomic distances ( $\AA$ ) and angles (degrees) for (a) $\mathrm{L}_{-} \mathrm{CuC}_{4} \mathrm{H}_{4} \mathrm{O}_{6} \cdot 3 \mathrm{H}_{2} \mathrm{O}$ and (b) DL- $\mathrm{CuC}_{4} \mathrm{H}_{4} \mathrm{O}_{6} \cdot 2 \mathrm{H}_{2} \mathrm{O}$

\begin{tabular}{|c|c|c|c|}
\hline \multicolumn{4}{|c|}{ (a) $\mathrm{L}-\mathrm{CuC}_{4} \mathrm{H}_{4} \mathrm{O}_{6} \cdot 3 \mathrm{H}_{2} \mathrm{O}$} \\
\hline $\mathrm{Cu}(1)-\mathrm{O}(2)$ & $1.936(2)$ & $\mathrm{Cu}(1)-\mathrm{O}(3)$ & $2.014(2)$ \\
\hline $\mathrm{Cu}(1)-\mathrm{O}(10)^{(1)}$ & $2.437(2)$ & $\mathrm{Cu}(1)-\mathrm{O}(11)^{(1)}$ & $1.920(1)$ \\
\hline $\mathrm{Cu}(1)-\mathrm{O}(12)^{(2)}$ & $2.342(2)$ & $\mathrm{Cu}(1)-\mathrm{O}(14)^{(3)}$ & $1.974(2)$ \\
\hline $\mathrm{Cu}(2)-\mathrm{O}(4)$ & $2.398(1)$ & $\mathrm{Cu}(2)-\mathrm{O}(5)$ & $1.928(1)$ \\
\hline $\mathrm{Cu}(2)-\mathrm{O}(7)$ & $1.976(1)$ & $\mathrm{Cu}(2)-\mathrm{O}(8)^{(1)}$ & $1.938(1)$ \\
\hline $\mathrm{Cu}(2)-\mathrm{O}(9)^{(1)}$ & $2.033(1)$ & $\mathrm{Cu}(2)-\mathrm{O}(13)$ & $2.382(2)$ \\
\hline $\mathrm{O}(1)-\mathrm{C}(1)$ & $1.239(2)$ & $\mathrm{O}(2)-\mathrm{C}(1)$ & $1.274(2)$ \\
\hline $\mathrm{O}(3)-\mathrm{C}(2)$ & $1.432(2)$ & $\mathrm{O}(4)-\mathrm{C}(3)$ & $1.426(2)$ \\
\hline $\mathrm{O}(5)-\mathrm{C}(4)$ & $1.279(2)$ & $\mathrm{O}(6)-\mathrm{C}(4)$ & $1.237(2)$ \\
\hline $\mathrm{O}(7)-\mathrm{C}(5)$ & $1.252(2)$ & $\mathrm{O}(8)-\mathrm{C}(5)$ & $1.256(2)$ \\
\hline $\mathrm{O}(9)-\mathrm{C}(6)$ & $1.429(2)$ & $\mathrm{O}(10)-\mathrm{C}(7)$ & $1.422(2)$ \\
\hline $\mathrm{O}(11)-\mathrm{C}(8)$ & $1.277(2)$ & $\mathrm{O}(12)-\mathrm{C}(8)$ & $1.240(2)$ \\
\hline $\mathrm{C}(1)-\mathrm{C}(2)$ & $1.529(3)$ & $\mathrm{C}(2)-\mathrm{C}(3)$ & $1.536(2)$ \\
\hline$C(3)-C(4)$ & $1.530(3)$ & $C(6)-C(5)$ & $1.537(2)$ \\
\hline$C(7)-C(6)$ & $1.530(3)$ & $\mathrm{C}(8)-\mathrm{C}(7)$ & $1.538(3)$ \\
\hline $\mathrm{O}(1)-\mathrm{C}(1)-\mathrm{O}(2)$ & $124.0(2)$ & $\mathrm{O}(1)-\mathrm{C}(1)-\mathrm{C}(2)$ & $118.3(2)$ \\
\hline $\mathrm{O}(2)-\mathrm{C}(1)-\mathrm{C}(2)$ & $117.7(2)$ & $\mathrm{O}(3)-\mathrm{C}(2)-\mathrm{C}(1)$ & $108.9(1)$ \\
\hline $\mathrm{O}(3)-\mathrm{C}(2)-\mathrm{C}(3)$ & $107.4(1)$ & $\mathrm{O}(4)-\mathrm{C}(3)-\mathrm{C}(4)$ & $112.4(1)$ \\
\hline $\mathrm{O}(4)-\mathrm{C}(3)-\mathrm{C}(2)$ & $110.4(1)$ & $\mathrm{O}(5)-\mathrm{C}(4)-\mathrm{O}(6)$ & $123.9(2)$ \\
\hline $\mathrm{O}(5)-\mathrm{C}(4)-\mathrm{C}(3)$ & $120.0(2)$ & $\mathrm{O}(6)-\mathrm{C}(4)-\mathrm{C}(3)$ & $116.0(2)$ \\
\hline $\mathrm{C}(1)-\mathrm{C}(2)-\mathrm{C}(3)$ & $110.2(2)$ & $\mathrm{C}(2)-\mathrm{C}(3)-\mathrm{C}(4)$ & $109.7(2)$ \\
\hline $\mathrm{O}(7)-\mathrm{C}(5)-\mathrm{O}(8)$ & $125.2(2)$ & $\mathrm{O}(7)-\mathrm{C}(5)-\mathrm{C}(6)$ & $116.7(2)$ \\
\hline $\mathrm{O}(8)-\mathrm{C}(5)-\mathrm{C}(6)$ & $118.1(2)$ & $\mathrm{O}(9)-\mathrm{C}(6)-\mathrm{C}(5)$ & $107.7(1)$ \\
\hline $\mathrm{O}(9)-\mathrm{C}(6)-\mathrm{C}(7)$ & $110.2(1)$ & $\mathrm{O}(10)-\mathrm{C}(7)-\mathrm{C}(6)$ & $110.0(1)$ \\
\hline $\mathrm{O}(10)-\mathrm{C}(7)-\mathrm{C}(8)$ & $111.9(1)$ & $\mathrm{O}(11)-\mathrm{C}(8)-\mathrm{O}(12)$ & $124.2(2)$ \\
\hline $\mathrm{O}(11)-\mathrm{C}(8)-\mathrm{C}(7)$ & $119.4(2)$ & $\mathrm{O}(12)-\mathrm{C}(8)-\mathrm{C}(7)$ & $116.2(2)$ \\
\hline$C(5)-C(6)-C(7)$ & $110.1(2)$ & $C(6)-C(7)-C(8)$ & $112.1(2)$ \\
\hline \multicolumn{4}{|c|}{ (b) $\mathrm{DL}-\mathrm{CuC}_{4} \mathrm{H}_{4} \mathrm{O}_{6} \cdot 2 \mathrm{H}_{2} \mathrm{O}$} \\
\hline $\mathrm{Cu}(1)-\mathrm{O}(2)$ & $1.936(2)$ & $\mathrm{Cu}(1)-\mathrm{O}(3)$ & $1.982(2)$ \\
\hline $\mathrm{Cu}(1)-\mathrm{O}(8)^{(4)}$ & $1.946(2)$ & $\mathrm{Cu}(1)-\mathrm{O}(9)^{(4)}$ & $2.338(2)$ \\
\hline $\mathrm{Cu}(1)-\mathrm{O}(12)^{(5)}$ & $1.936(2)$ & $\mathrm{Cu}(1)-\mathrm{O}(13)$ & $2.397(3)$ \\
\hline $\mathrm{Cu}(2)-\mathrm{O}(1)^{(6)}$ & $2.263(2)$ & $\mathrm{Cu}(2)-\mathrm{O}(4)$ & $2.058(2)$ \\
\hline $\mathrm{Cu}(2)-\mathrm{O}(5)$ & $1.922(2)$ & $\mathrm{Cu}(2)-\mathrm{O}(10)^{(4)}$ & $2.421(2)$ \\
\hline $\mathrm{Cu}(2)-\mathrm{O}(11)^{(4)}$ & $1.952(2)$ & $\mathrm{Cu}(2)-\mathrm{O}(16)$ & $2.005(2)$ \\
\hline $\mathrm{O}(1)-\mathrm{C}(1)$ & $1.252(3)$ & $\mathrm{O}(2)-\mathrm{C}(1)$ & $1.265(3)$ \\
\hline $\mathrm{O}(3)-\mathrm{C}(2)$ & $1.426(3)$ & $\mathrm{O}(4)-\mathrm{C}(3)$ & $1.440(3)$ \\
\hline $\mathrm{O}(5)-\mathrm{C}(4)$ & $1.273(3)$ & $\mathrm{O}(6)-\mathrm{C}(4)$ & $1.245(3)$ \\
\hline $\mathrm{O}(7)-\mathrm{C}(5)$ & $1.251(3)$ & $\mathrm{O}(8)-\mathrm{C}(5)$ & $1.272(3)$ \\
\hline $\mathrm{O}(9)-\mathrm{C}(6)$ & $1.430(3)$ & $\mathrm{O}(10)-\mathrm{C}(7)$ & $1.418(3)$ \\
\hline $\mathrm{O}(11)-\mathrm{C}(8)$ & $1.261(3)$ & $\mathrm{O}(12)-\mathrm{C}(8)$ & $1.260(3)$ \\
\hline$C(1)-C(2)$ & $1.535(4)$ & $\mathrm{C}(2)-\mathrm{C}(3)$ & $1.530(4)$ \\
\hline$C(3)-C(4)$ & $1.525(4)$ & $C(5)-C(6)$ & $1.528(4)$ \\
\hline$C(6)-C(7)$ & $1.531(4)$ & $C(7)-C(8)$ & $1.532(4)$ \\
\hline $\mathrm{O}(1)-\mathrm{C}(1)-\mathrm{O}(2)$ & $124.5(2)$ & $\mathrm{O}(1)-\mathrm{C}(1)-\mathrm{C}(2)$ & $118.0(2)$ \\
\hline $\mathrm{O}(2)-\mathrm{C}(1)-\mathrm{C}(2)$ & $117.5(2)$ & $\mathrm{O}(3)-\mathrm{C}(2)-\mathrm{C}(1)$ & $107.2(2)$ \\
\hline $\mathrm{O}(3)-\mathrm{C}(2)-\mathrm{C}(3)$ & $111.5(2)$ & $\mathrm{O}(4)-\mathrm{C}(3)-\mathrm{C}(2)$ & $111.5(2)$ \\
\hline $\mathrm{O}(4)-\mathrm{C}(3)-\mathrm{C}(4)$ & $110.2(2)$ & $\mathrm{O}(5)-\mathrm{C}(4)-\mathrm{O}(6)$ & $124.0(2)$ \\
\hline $\mathrm{O}(5)-\mathrm{C}(4)-\mathrm{C}(3)$ & $118.1(2)$ & $\mathrm{O}(6)-\mathrm{C}(4)-\mathrm{C}(3)$ & $117.8(2)$ \\
\hline $\mathrm{C}(1)-\mathrm{C}(2)-\mathrm{C}(3)$ & $109.6(2)$ & $\mathrm{C}(2)-\mathrm{C}(3)-\mathrm{C}(4)$ & $113.9(2)$ \\
\hline $\mathrm{O}(7)-\mathrm{C}(5)-\mathrm{O}(8)$ & $123.4(2)$ & $\mathrm{O}(7)-\mathrm{C}(5)-\mathrm{C}(6)$ & $115.8(2)$ \\
\hline $\mathrm{O}(8)-\mathrm{C}(5)-\mathrm{C}(6)$ & $120.8(2)$ & $\mathrm{O}(9)-\mathrm{C}(6)-\mathrm{C}(5)$ & $112.1(2)$ \\
\hline $\mathrm{O}(9)-\mathrm{C}(6)-\mathrm{C}(7)$ & $108.5(2)$ & $\mathrm{O}(10)-\mathrm{C}(7)-\mathrm{C}(6)$ & $107.7(2)$ \\
\hline $\mathrm{O}(10)-\mathrm{C}(7)-\mathrm{C}(8)$ & $111.9(2)$ & $\mathrm{O}(11)-\mathrm{C}(8)-\mathrm{O}(12)$ & $125.2(2)$ \\
\hline $\mathrm{O}(11)-\mathrm{C}(8)-\mathrm{C}(7)$ & $121.3(2)$ & $\mathrm{O}(12)-\mathrm{C}(8)-\mathrm{C}(7)$ & $113.5(2)$ \\
\hline$C(5)-C(6)-C(7)$ & $111.5(2)$ & $C(6)-C(7)-C(8)$ & $106.3(2)$ \\
\hline
\end{tabular}


Symmetry codes: (1) $-x+1, y+1 / 2,-z+1$; (2) $x-1, y, z-1$; (3) $x, y, z-1$; (4) $x+1, y, z$; (5) $-x+1, y-1 / 2,-z+1 / 2$; (6) $-x+1$, $y+1 / 2,-z+1 / 2$.

The relevant crystal data, and a summary of intensity data collection and structure refinement are given in Table 1. Figure 2 shows the projections of the (a) $\mathrm{L}-\mathrm{CuC}_{4} \mathrm{H}_{4} \mathrm{O}_{6} \cdot 3 \mathrm{H}_{2} \mathrm{O}$ and (b) DL- $\mathrm{CuC}_{4} \mathrm{H}_{4} \mathrm{O}_{6} \cdot 2 \mathrm{H}_{2} \mathrm{O}$ crystal structures along the $a$-axis. The positional parameters in fractions of the unit cell and the thermal parameters are listed in Table 2. Selected bond lengths and angles are given in Table 3, and hydrogen-bond geometries are presented in Table 4.

\subsection{Structure Description}

The observed lattice constants of $\mathrm{L}-\mathrm{CuC}_{4} \mathrm{H}_{4} \mathrm{O}_{6} \cdot 3 \mathrm{H}_{2} \mathrm{O}$ are close to those observed by Labutina et al. (Labutina, Marychev, Portnov, Somov, \& Chuprunov, 2011) and Soylu (Soylu, 1996), but differ from those of copper tartrate crystals reported in the previous papers (Binitha \& Pradyumnan, 2013; Bridle \& Lomer, 1965; Jethva, Dabhi, \& Joshi, 2016). The discrepancies are probably mainly attributed to the difference in number of bound water molecules, as described in Introduction. In this study, the unit cell structure of $\mathrm{L}-\mathrm{CuC}_{4} \mathrm{H}_{4} \mathrm{O}_{6} \cdot 3 \mathrm{H}_{2} \mathrm{O}$ consists of two non-equivalent $\mathrm{Cu}$ atoms, two crystallographically independent $\mathrm{C}_{4} \mathrm{H}_{4} \mathrm{O}_{6}$ molecules, and six independent $\mathrm{H}_{2} \mathrm{O}$ molecules. Figure 2(a) shows that the $\mathrm{Cu}$ atoms and $\mathrm{C}_{4} \mathrm{H}_{4} \mathrm{O}_{6}$ molecules are periodically arranged along the $b$ - and $c$-axes. Moreover, the $\mathrm{Cu}$ atoms are bonded to six nearest-neighboring $\mathrm{O}$ atoms, forming slightly distorted $\mathrm{CuO}_{6}$ octahedra, as listed in Table 3(a). These six $\mathrm{O}$ atoms include five $\mathrm{O}$ atoms from three $\mathrm{C}_{4} \mathrm{H}_{4} \mathrm{O}_{6}$ molecules and one $\mathrm{O}$ atom from the $\mathrm{H}_{2} \mathrm{O}$ molecule. Thus, the three $\mathrm{C}_{4} \mathrm{H}_{4} \mathrm{O}_{6}$ and one $\mathrm{H}_{2} \mathrm{O}$ molecules are connected to each other through the $\mathrm{Cu}-\mathrm{O}$ bonds. The lengths of the $\mathrm{Cu}-\mathrm{O}$ bonds are in the range of 1.920(1)-2.437(2) $\AA$, and the average $\mathrm{Cu}-\mathrm{O}$ distance is $2.107 \AA$. As shown in Table 4 and Fig. 2(a), the $\mathrm{C}_{4} \mathrm{H}_{4} \mathrm{O}_{6}$ molecules are connected each other by four $\mathrm{C}-\mathrm{H}-\mathrm{O}$ and $\mathrm{O}(4)-\mathrm{H}-\mathrm{O}(10)$ hydrogen bonds, and zigzag hydrogen-bonded chains are formed along the $b$-axis. Moreover, hydrogen-bonding networks consisting of $\mathrm{C}_{4} \mathrm{H}_{4} \mathrm{O}_{6}$ and one or two $\mathrm{H}_{2} \mathrm{O}$ molecules connected by other $\mathrm{O}-\mathrm{H}-\mathrm{O}$ bonds are present along the $a$-axis. No hydrogen-bonded chains exist along the $c$-axis; however, there are $\mathrm{C}_{4} \mathrm{H}_{4} \mathrm{O}_{6}-\mathrm{Cu}-\mathrm{C}_{4} \mathrm{H}_{4} \mathrm{O}_{6}$ chains running along the $c$-axis linked by the $\mathrm{Cu}-\mathrm{O}$ bonds mentioned above.

On the other hand, the unit cell structure of DL-CuC $\mathrm{H}_{4} \mathrm{O}_{6} \cdot 2 \mathrm{H}_{2} \mathrm{O}$ consists of two non-equivalent $\mathrm{Cu}$ atoms, two crystallographically independent $\mathrm{C}_{4} \mathrm{H}_{4} \mathrm{O}_{6}$ molecules, and four independent $\mathrm{H}_{2} \mathrm{O}$ molecules. Structural differences between the $\mathrm{L}-\mathrm{CuC}_{4} \mathrm{H}_{4} \mathrm{O}_{6} \cdot 3 \mathrm{H}_{2} \mathrm{O}$ and DL- $\mathrm{CuC}_{4} \mathrm{H}_{4} \mathrm{O}_{6} \cdot 2 \mathrm{H}_{2} \mathrm{O}$ crystals are as follows: the size of the unit cell of the DL- $\mathrm{CuC}_{4} \mathrm{H}_{4} \mathrm{O}_{6} \cdot 2 \mathrm{H}_{2} \mathrm{O}$ crystal is approximately twice that of the $\mathrm{L}-\mathrm{CuC}_{4} \mathrm{H}_{4} \mathrm{O}_{6} \cdot 3 \mathrm{H}_{2} \mathrm{O}$ crystal, and the number of molecules per unit cell becomes doubled, as given in Table 1. Furthermore, the number of water molecules in the formula unit reduces from three to two. Figure 2(b) shows that the $\mathrm{Cu}$ atoms are periodically arranged along the $b$ - and $c$-axes, and the atoms and $\mathrm{C}_{4} \mathrm{H}_{4} \mathrm{O}_{6}$ molecules are located around eight $\mathrm{H}_{2} \mathrm{O}$ molecules near the center of the $b c$-plane. As listed in Table 3(b), the $\mathrm{Cu}$ atoms are bonded to six nearest-neighboring $\mathrm{O}$ atoms, forming slightly distorted $\mathrm{CuO}_{6}$ octahedra, similarly to that in $\mathrm{L}-\mathrm{CuC}_{4} \mathrm{H}_{4} \mathrm{O}_{6} \cdot 3 \mathrm{H}_{2} \mathrm{O}$. The six atoms bonding to the $\mathrm{Cu}$ atoms are five $\mathrm{O}$ atoms from three $\mathrm{C}_{4} \mathrm{H}_{4} \mathrm{O}_{6}$ molecules and one $\mathrm{O}$ atom from the $\mathrm{H}_{2} \mathrm{O}$ molecule. Therefore, the three $\mathrm{C}_{4} \mathrm{H}_{4} \mathrm{O}_{6}$ and one $\mathrm{H}_{2} \mathrm{O}$ molecules are connected to each other through $\mathrm{Cu}-\mathrm{O}$ bonds. The lengths of the $\mathrm{Cu}-\mathrm{O}$ bonds are in the range of 1.922(2) - 2.421(2) $\AA$, and the average $\mathrm{Cu}-\mathrm{O}$ distance is $2.096 \AA$. Since the average distance is slightly smaller than that of $\mathrm{L}-\mathrm{CuC}_{4} \mathrm{H}_{4} \mathrm{O}_{6} \cdot 3 \mathrm{H}_{2} \mathrm{O}(2.107$ $\AA$ ), the size of the $\mathrm{CuO}_{6}$ octahedra in DL-CuC $\mathrm{Cu}_{4} \mathrm{O}_{6} \cdot 2 \mathrm{H}_{2} \mathrm{O}$ is slightly smaller than that in $\mathrm{L}-\mathrm{CuC}_{4} \mathrm{H}_{4} \mathrm{O}_{6} \cdot 3 \mathrm{H}_{2} \mathrm{O}$. Four $\mathrm{C}_{4} \mathrm{H}_{4} \mathrm{O}_{6}$ molecules located near the ac-plane are connected to each other by four $\mathrm{C}-\mathrm{H}-\mathrm{O}$ and three $\mathrm{O}-\mathrm{H}-\mathrm{O}$ hydrogen bonds, and zigzag hydrogen-bonded chains consisting of the molecules are running along the $a$-axis. Furthermore, hydrogen-bonded chains formed by the $\mathrm{C}_{4} \mathrm{H}_{4} \mathrm{O}_{6}$ and two $\mathrm{H}_{2} \mathrm{O}$ molecules connected through the $\mathrm{O}-\mathrm{H}-\mathrm{O}$ bonds are present along the $b$ - and $c$-axes, and $\mathrm{C}_{4} \mathrm{H}_{4} \mathrm{O}_{6}-\mathrm{Cu}-\mathrm{C}_{4} \mathrm{H}_{4} \mathrm{O}_{6}$ chains formed by the $\mathrm{Cu}-\mathrm{O}$ bonds mentioned above are running along the $c$-axis.

The lengths of six $\mathrm{O}-\mathrm{C}$ and four $\mathrm{C}-\mathrm{C}$ bonds in the $\mathrm{C}_{4} \mathrm{H}_{4} \mathrm{O}_{6}$ molecules of $\mathrm{L}-\mathrm{CuC}_{4} \mathrm{H}_{4} \mathrm{O}_{6} \cdot 3 \mathrm{H}_{2} \mathrm{O}$ and DL- $\mathrm{CuC}_{4} \mathrm{H}_{4} \mathrm{O}_{6} \cdot 2 \mathrm{H}_{2} \mathrm{O}$ are, respectively, very similar to those of other tartrate crystals in our previous studies (Fukami, Hiyajyo, Tahara, \& Yasuda, 2017; Fukami \& Tahara, 2018; Fukami \& Tahara, 2020). A comparison of these bond lengths reveals that the two $\mathrm{O}-\mathrm{C}$ bonds of hydroxyl groups in both crystals have single-bond character, and the remaining four bonds have double-bond character; all the $\mathrm{C}-\mathrm{C}$ bonds have single-bond character. The angles between the two least-squares planes of atoms, $[\mathrm{C}(1) \mathrm{C}(2) \mathrm{O}(1) \mathrm{O}(2) \mathrm{O}(3)$ and $\mathrm{C}(3) \mathrm{C}(4) \mathrm{O}(4) \mathrm{O}(5) \mathrm{O}(6)]$, and $[\mathrm{C}(5) \mathrm{C}(6) \mathrm{O}(7) \mathrm{O}(8) \mathrm{O}(9)$ and $\mathrm{C}(7) \mathrm{C}(8) \mathrm{O}(10) \mathrm{O}(11) \mathrm{O}(12)]$, in the $\mathrm{C}_{4} \mathrm{H}_{4} \mathrm{O}_{6}$ molecules of $\mathrm{L}^{-} \mathrm{CuC}_{4} \mathrm{H}_{4} \mathrm{O}_{6} \cdot 3 \mathrm{H}_{2} \mathrm{O}$ were $48.73(7)^{\circ}$ and $56.85(6)^{\circ}$, respectively. The angles between these planes in DL- $\mathrm{CuC}_{4} \mathrm{H}_{4} \mathrm{O}_{6} \cdot 2 \mathrm{H}_{2} \mathrm{O}$ were $64.39(4)^{\circ}$ and $58.46(8)^{\circ}$, respectively. The angles in DL- $\mathrm{CuC}_{4} \mathrm{H}_{4} \mathrm{O}_{6} \cdot 2 \mathrm{H}_{2} \mathrm{O}$ were approximately $9^{\circ}$ larger than those in $\mathrm{L}-\mathrm{CuC}_{4} \mathrm{H}_{4} \mathrm{O}_{6} \cdot 3 \mathrm{H}_{2} \mathrm{O}$, comparing the closing values of the angle. 
Table 4. Hydrogen bond distances ( $\AA$ ) and angles (degrees) for (a) $\mathrm{L}-\mathrm{CuC}_{4} \mathrm{H}_{4} \mathrm{O}_{6} \cdot 3 \mathrm{H}_{2} \mathrm{O}$ and (b) DL-CuC $\mathrm{H}_{4} \mathrm{O}_{6} \cdot 2 \mathrm{H}_{2} \mathrm{O}$

\begin{tabular}{|c|c|c|c|c|}
\hline \multicolumn{5}{|l|}{ (a) $\mathrm{L}-\mathrm{CuC}_{4} \mathrm{H}_{4} \mathrm{O}_{6} \cdot 3 \mathrm{H}_{2} \mathrm{O}$} \\
\hline $\mathrm{D}-\mathrm{H} \cdots \mathrm{A}$ & $\mathrm{D}-\mathrm{H}$ & $\mathrm{H} \cdots \mathrm{A}$ & $\mathrm{D} \cdots \mathrm{A}$ & $<\mathrm{D}-\mathrm{H} \cdots \mathrm{A}$ \\
\hline $\mathrm{C}(2)-\mathrm{H}(1) \cdots \mathrm{O}(12)^{(1)}$ & $0.92(3)$ & $2.54(3)$ & $3.420(2)$ & $161(2)$ \\
\hline $\mathrm{C}(3)-\mathrm{H}(2) \cdots \mathrm{O}(7)^{(1)}$ & $0.93(3)$ & $2.51(3)$ & $3.377(2)$ & $156(2)$ \\
\hline $\mathrm{C}(6)-\mathrm{H}(3) \cdots \mathrm{O}(6)^{(2)}$ & $0.95(3)$ & $2.40(3)$ & $3.286(3)$ & $156(2)$ \\
\hline $\mathrm{C}(7)-\mathrm{H}(4) \cdots \mathrm{O}(1)^{(2)}$ & $0.89(3)$ & $2.56(3)$ & $3.355(2)$ & $148(2)$ \\
\hline $\mathrm{O}(3)-\mathrm{H}(5) \cdots \mathrm{O}(17)^{(3)}$ & $0.71(3)$ & $2.01(3)$ & $2.704(3)$ & $166(4)$ \\
\hline $\mathrm{O}(4)-\mathrm{H}(6) \cdots \mathrm{O}(10)^{(2)}$ & $0.79(4)$ & $1.99(3)$ & $2.745(2)$ & $160(4)$ \\
\hline $\mathrm{O}(9)-\mathrm{H}(7) \cdots \mathrm{O}(18)^{(4)}$ & $0.94(4)$ & $1.64(4)$ & $2.567(2)$ & $168(4)$ \\
\hline $\mathrm{O}(10)-\mathrm{H}(8) \cdots \mathrm{O}(15)$ & $0.75(4)$ & $1.92(4)$ & $2.660(3)$ & $172(4)$ \\
\hline $\mathrm{O}(13)-\mathrm{H}(9) \cdots \mathrm{O}(6)^{(5)}$ & $0.97(4)$ & $1.73(4)$ & $2.698(3)$ & $171(4)$ \\
\hline $\mathrm{O}(13)-\mathrm{H}(10) \cdots \mathrm{O}(15)$ & $0.61(5)$ & $2.85(5)$ & $3.410(5)$ & $156(6)$ \\
\hline $\mathrm{O}(14)-\mathrm{H}(11) \cdots \mathrm{O}(17)^{(2)}$ & $0.87(3)$ & $1.84(3)$ & $2.707(3)$ & $178(3)$ \\
\hline $\mathrm{O}(14)-\mathrm{H}(12) \cdots \mathrm{O}(1)^{(2)}$ & $0.72(4)$ & $2.03(4)$ & $2.753(3)$ & $176(4)$ \\
\hline $\mathrm{O}(15)-\mathrm{H}(13) \cdots \mathrm{O}(17)^{(1)}$ & $0.97(5)$ & $2.70(5)$ & $3.636(5)$ & $161(4)$ \\
\hline $\mathrm{O}(15)-\mathrm{H}(14) \cdots \mathrm{O}(2)^{(6)}$ & $0.82(4)$ & $2.14(4)$ & $2.808(3)$ & $139(4)$ \\
\hline $\mathrm{O}(15)-\mathrm{H}(14) \cdots \mathrm{O}(14)$ & $0.82(4)$ & $2.61(5)$ & $3.314(4)$ & $145(4)$ \\
\hline $\mathrm{O}(16)-\mathrm{H}(15) \cdots \mathrm{O}(8)$ & $0.78(5)$ & $2.93(5)$ & $3.548(3)$ & $138(4)$ \\
\hline $\mathrm{O}(16)-\mathrm{H}(16) \cdots \mathrm{O}(13)^{(1)}$ & $0.76(4)$ & $2.22(4)$ & $2.816(3)$ & $135(4)$ \\
\hline $\mathrm{O}(17)-\mathrm{H}(17) \cdots \mathrm{O}(16)$ & $0.97(5)$ & $1.83(5)$ & $2.804(4)$ & $177(4)$ \\
\hline $\mathrm{O}(17)-\mathrm{H}(18) \cdots \mathrm{O}(1)^{(7)}$ & $0.76(5)$ & $2.24(4)$ & $2.950(3)$ & $156(4)$ \\
\hline $\mathrm{O}(18)-\mathrm{H}(19) \cdots \mathrm{O}(16)$ & $0.69(4)$ & $2.11(4)$ & $2.762(3)$ & $159(5)$ \\
\hline $\mathrm{O}(18)-\mathrm{H}(20) \cdots \mathrm{O}(5)^{(2)}$ & $1.07(4)$ & $1.75(4)$ & $2.752(2)$ & $155(3)$ \\
\hline \multicolumn{5}{|l|}{ (b) $\mathrm{DL}-\mathrm{CuC}_{4} \mathrm{H}_{4} \mathrm{O}_{6} \cdot 2 \mathrm{H}_{2} \mathrm{O}$} \\
\hline $\mathrm{C}(2)-\mathrm{H}(1) \cdots \mathrm{O}(7)$ & $0.95(5)$ & $2.70(5)$ & $3.588(3)$ & $156(4)$ \\
\hline $\mathrm{C}(3)-\mathrm{H}(2) \cdots \mathrm{O}(12)$ & $0.89(5)$ & $2.49(5)$ & $3.328(3)$ & $156(4)$ \\
\hline $\mathrm{C}(6)-\mathrm{H}(3) \cdots \mathrm{O}(1)$ & $0.93(4)$ & $2.46(4)$ & $3.375(3)$ & $165(4)$ \\
\hline $\mathrm{C}(7)-\mathrm{H}(4) \cdots \mathrm{O}(6)$ & $0.95(5)$ & $2.46(5)$ & $3.390(3)$ & $165(4)$ \\
\hline $\mathrm{O}(3)-\mathrm{H}(5) \cdots \mathrm{O}(6)^{(8)}$ & $0.87(7)$ & $1.70(7)$ & $2.560(3)$ & $166(7)$ \\
\hline $\mathrm{O}(4)-\mathrm{H}(6) \cdots \mathrm{O}(9)^{(9)}$ & $0.59(7)$ & $2.22(7)$ & $2.771(3)$ & $157(8)$ \\
\hline $\mathrm{O}(9)-\mathrm{H}(7) \cdots \mathrm{O}(15)^{(10)}$ & $0.68(6)$ & $2.08(6)$ & $2.754(3)$ & $173(7)$ \\
\hline $\mathrm{O}(10)-\mathrm{H}(8) \cdots \mathrm{O}(7)^{(11)}$ & $0.65(5)$ & $2.05(5)$ & $2.692(3)$ & $171(6)$ \\
\hline $\mathrm{O}(13)-\mathrm{H}(9) \cdots \mathrm{O}(1)^{(12)}$ & $0.66(7)$ & $2.31(7)$ & $2.901(3)$ & $151(7)$ \\
\hline $\mathrm{O}(13)-\mathrm{H}(10) \cdots \mathrm{O}(14)^{(13)}$ & $0.73(7)$ & $2.17(7)$ & $2.881(5)$ & $165(7)$ \\
\hline $\mathrm{O}(14)-\mathrm{H}(11) \cdots \mathrm{O}(5)^{(12)}$ & $0.77(8)$ & $2.39(8)$ & $3.136(5)$ & $166(8)$ \\
\hline $\mathrm{O}(14)-\mathrm{H}(12) \cdots \mathrm{O}(13)^{(14)}$ & $1.17(9)$ & $1.97(9)$ & $3.108(6)$ & $162(7)$ \\
\hline $\mathrm{O}(15)-\mathrm{H}(13) \cdots \mathrm{O}(8)^{(14)}$ & $0.86(5)$ & $2.06(5)$ & $2.922(3)$ & $178(5)$ \\
\hline $\mathrm{O}(15)-\mathrm{H}(14) \cdots \mathrm{O}(14)$ & $0.86(7)$ & $2.03(7)$ & $2.878(4)$ & $173(6)$ \\
\hline $\mathrm{O}(16)-\mathrm{H}(15) \cdots \mathrm{O}(15)^{(15)}$ & $0.87(5)$ & $1.86(6)$ & $2.729(3)$ & $173(5)$ \\
\hline $\mathrm{O}(16)-\mathrm{H}(16) \cdots \mathrm{O}(7)^{(8)}$ & $0.93(6)$ & $1.76(6)$ & $2.676(3)$ & $166(5)$ \\
\hline
\end{tabular}

Symmetry codes: (1) $-x+1, y-1 / 2,-z+1$; (2) $-x+1, y+1 / 2,-z+1$; (3) $x-1, y, z$; (4) $-x+2, y-1 / 2,-z+1$; (5) $-x, y+1 / 2,-z+1$; (6) $x, y, z+1$; (7) $-x+1, y+1 / 2,-z$; (8) $-x+1,-y+1,-z$; (9) $x+1, y, z$; (10) $x-1, y, z$; (11) $-x,-y+1,-z$; (12) $-x+1, y-1 / 2,-$ $z+1 / 2$; (13) $x,-y+1 / 2, z-1 / 2$; (14) $-x+1, y+1 / 2,-z+1 / 2$; (15) $-x+2, y+1 / 2,-z+1 / 2$.

\subsection{Thermal Analysis}

Figure 3 shows the TG, differential TG (DTG), and DTA curves in the temperature range of 300-1250 K for the $\mathrm{L}-\mathrm{CuC}_{4} \mathrm{H}_{4} \mathrm{O}_{6} \cdot 3 \mathrm{H}_{2} \mathrm{O}$ and DL- $\mathrm{CuC}_{4} \mathrm{H}_{4} \mathrm{O}_{6} \cdot 2 \mathrm{H}_{2} \mathrm{O}$ crystals. The sample weights (powder) of $\mathrm{L}-\mathrm{CuC}_{4} \mathrm{H}_{4} \mathrm{O}_{6} \cdot 3 \mathrm{H}_{2} \mathrm{O}$ and DL- $\mathrm{CuC}_{4} \mathrm{H}_{4} \mathrm{O}_{6} \cdot 2 \mathrm{H}_{2} \mathrm{O}$ were 6.59 and $7.81 \mathrm{mg}$, respectively. The heating rate was $10 \mathrm{~K} \mathrm{~min}^{-1}$ under a dry nitrogen gas flow of $300 \mathrm{ml} \mathrm{min}^{-1}$. The observed TG curve of $\mathrm{L}-\mathrm{CuC}_{4} \mathrm{H}_{4} \mathrm{O}_{6} \cdot 3 \mathrm{H}_{2} \mathrm{O}$ is remarkably similar to those in the previous papers (Binitha \& Pradyumnan, 2013; Jethva, Dabhi, \& Joshi, 2016). The DTA curve of L-CuC $\mathrm{CH}_{4} \mathrm{O}_{6} \cdot 3 \mathrm{H}_{2} \mathrm{O}$ shows three endothermic peaks at 327,527 , and $580 \mathrm{~K}$ including a small peak, and the DTG curve shows three peaks at 323, 531, and $573 \mathrm{~K}$. On the other hand, three endothermic peaks at 380,524 , and $558 \mathrm{~K}$ are observed in the DTA curve of DL- $\mathrm{CuC}_{4} \mathrm{H}_{4} \mathrm{O}_{6} \cdot 2 \mathrm{H}_{2} \mathrm{O}$, and three peaks at 381,527 , and $536 \mathrm{~K}$ are observed in the corresponding DTG curve. The 
endothermic peak temperatures on the DTA curves of both crystals are, respectively, very close to those on the DTG curves. The DTG curve, which is the first derivative of TG curve, reveals the temperature dependence of weight loss rate due to thermal decomposition of sample. Thus, the DTA peaks are associated with the maximum rate of weight loss in the TG curve. DSC measurements on the powder samples of both crystals were performed in the temperature range from 100 to $310 \mathrm{~K}$ at a heating rate of $10 \mathrm{~K} \mathrm{~min}^{-1}$. No obvious endothermic or exothermic peaks were observed in the DSC curves in this temperature range, except for gentle changes in slope of the baseline in the curves due to the endothermic peak at 327 or $380 \mathrm{~K}$. In general, it is believed that a clear peak in DSC curve is attributed to the change in exchange energy at phase transition. Thus, the obtained results indicate that there is no phase transition in the temperature range of $100-310 \mathrm{~K}$ in both the crystals.

Two large and two small weight losses are seen at around 350,500,600, and above $640 \mathrm{~K}$ in the TG curves for both crystals, as shown in Fig. 3(a) and (b). The experimental weight losses for $\mathrm{L}_{-} \mathrm{CuC}_{4} \mathrm{H}_{4} \mathrm{O}_{6} \cdot 3 \mathrm{H}_{2} \mathrm{O}$ in the temperature ranges of 300-460, 460-584, 584-640, and 640-1250 K were found to be 15.9, 52.1, 2.7 and 4.2\%, respectively, and those for DL- $\mathrm{CuC}_{4} \mathrm{H}_{4} \mathrm{O}_{6} \cdot 2 \mathrm{H}_{2} \mathrm{O}$ in the ranges of $300-460,460-546,546-640$, and $640-1250 \mathrm{~K}$ were 13.1, 45.5, 8.7 and $6.1 \%$, respectively. Table 5 shows the experimental and theoretical weight losses in each temperature range. The theoretical weight losses were calculated based on the following considerations.

Table 5. TG results for thermal decomposition of (a) $\mathrm{L}-\mathrm{CuC}_{4} \mathrm{H}_{4} \mathrm{O}_{6} \cdot 3 \mathrm{H}_{2} \mathrm{O}$ and (b) DL- $\mathrm{CuC}_{4} \mathrm{H}_{4} \mathrm{O}_{6} \cdot 2 \mathrm{H}_{2} \mathrm{O}$

\begin{tabular}{llll}
\hline \multicolumn{2}{l}{ (a) $\mathrm{L}-\mathrm{CuC}_{4} \mathrm{H}_{4} \mathrm{O}_{6} \cdot 3 \mathrm{H}_{2} \mathrm{O}$} & & \\
\hline Temp. range [K] & Weight loss (obs.) [\%] & Weight loss (cal.) [\%] & Elimination molecules \\
\hline $300-460$ & 15.9 & 17.0 & $5 \mathrm{H}_{2} \mathrm{O}$ \\
$460-584$ & 52.1 & 50.1 & $4 \mathrm{H}_{2} \mathrm{CO}, 4 \mathrm{CO},(1 / 2) \mathrm{O}_{2}, \mathrm{H}_{2} \mathrm{O}$ \\
$584-640$ & 2.7 & 3.0 & $(1 / 2) \mathrm{O}_{2}$ \\
$640-1250$ & 4.2 & 3.0 & $(1 / 2) \mathrm{O}_{2}$ \\
Total & 74.9 & 73.1 & \\
\hline (b) DL-CuC $\mathrm{H}_{4} \mathrm{O}_{6} \cdot 2 \mathrm{H}_{2} \mathrm{O}$ & & \\
\hline $300-460$ & 13.1 & 14.5 & $4 \mathrm{H}_{2} \mathrm{O}$ \\
$460-546$ & 45.5 & 41.2 & $4 \mathrm{H}_{2} \mathrm{CO}, 3 \mathrm{CO}$ \\
$546-640$ & 8.7 & 8.9 & $\mathrm{CO},(1 / 2) \mathrm{O}_{2}$ \\
$640-1250$ & 6.1 & 6.5 & $\mathrm{O}_{2}$ \\
Total & 73.4 & 71.1 & \\
\hline
\end{tabular}

The weight losses in the TG curves for both crystals may have been caused by the evolution of gases from the samples, similarly to our previous studies (Fukami, Hiyajyo, Tahara, \& Yasuda, 2017; Fukami \& Tahara, 2018; Fukami \& Tahara, 2020). There are two crystallographically independent formula units in the unit cells of both crystals, as described in Table 2(a) and (b). Therefore, the theoretical weight loss rates due to the thermal decomposition are calculated using twice the formula weight of $\mathrm{L}-\mathrm{CuC}_{4} \mathrm{H}_{4} \mathrm{O}_{6} \cdot 3 \mathrm{H}_{2} \mathrm{O}\left(2 \mathrm{M}_{\mathrm{L}}=531.33 \mathrm{~g} \mathrm{~mol}^{-1}\right)$ and of DL- $\mathrm{CuC}_{4} \mathrm{H}_{4} \mathrm{O}_{6} \cdot 2 \mathrm{H}_{2} \mathrm{O}\left(2 M_{\mathrm{DL}}=495.30 \mathrm{~g}\right.$ $\mathrm{mol}^{-1}$ ). The elimination of bound water molecules from the crystals presumably occurs with increasing temperature in the temperature range from 300 to $460 \mathrm{~K}$. Above the temperature of $460 \mathrm{~K}$, the evolutions of gases and copper compounds occur through chemical reactions described by the following chemical equations:

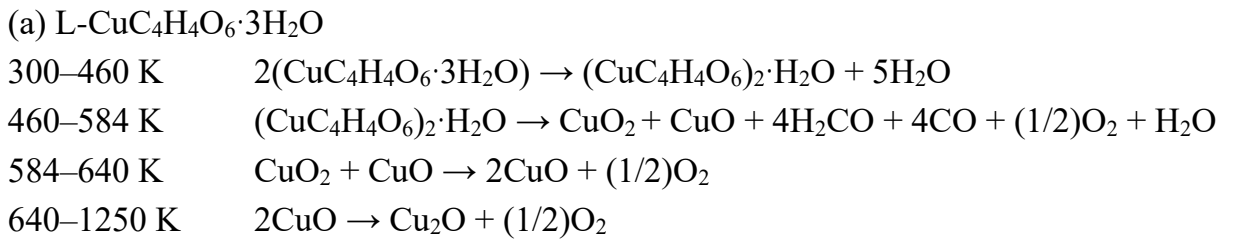

(b) $\mathrm{DL}-\mathrm{CuC}_{4} \mathrm{H}_{4} \mathrm{O}_{6} \cdot 2 \mathrm{H}_{2} \mathrm{O}$

$$
\begin{array}{ll}
300-460 \mathrm{~K} & 2\left(\mathrm{CuC}_{4} \mathrm{H}_{4} \mathrm{O}_{6} \cdot 2 \mathrm{H}_{2} \mathrm{O}\right) \rightarrow 2 \mathrm{CuC}_{4} \mathrm{H}_{4} \mathrm{O}_{6}+4 \mathrm{H}_{2} \mathrm{O} \\
460-546 \mathrm{~K} & 2 \mathrm{CuC}_{4} \mathrm{H}_{4} \mathrm{O}_{6} \rightarrow \mathrm{CuO}_{2}+\mathrm{CuCO}_{3}+4 \mathrm{H}_{2} \mathrm{CO}+3 \mathrm{CO} \\
546-640 \mathrm{~K} & \mathrm{CuO}_{2}+\mathrm{CuCO}_{3} \rightarrow \mathrm{CuO}_{2}+\mathrm{CuO}+\mathrm{CO}+(1 / 2) \mathrm{O}_{2} \\
640-1250 \mathrm{~K} & \mathrm{CuO}_{2}+\mathrm{CuO} \rightarrow \mathrm{Cu}_{2} \mathrm{O}+\mathrm{O}_{2} .
\end{array}
$$

As shown in the chemical reaction equations for the temperature range of 300-460 K, the evaporation of five or four bound water molecules from within the crystals occurs with increased temperatures. Therefore, the theoretical weight losses for $\mathrm{L}-\mathrm{CuC}_{4} \mathrm{H}_{4} \mathrm{O}_{6} \cdot 3 \mathrm{H}_{2} \mathrm{O}$ and DL- $\mathrm{CuC}_{4} \mathrm{H}_{4} \mathrm{O}_{6} \cdot 2 \mathrm{H}_{2} \mathrm{O}$ due to the evaporation of $\mathrm{H}_{2} \mathrm{O}$ are calculated to be $17.0 \%$ 
$(=5 \times 18.02 / 531.33)$ and $14.5 \%(=4 \times 18.02 / 495.30)$, respectively. According to the second chemical equation for $\mathrm{L}-\mathrm{CuC}_{4} \mathrm{H}_{4} \mathrm{O}_{6} \cdot 3 \mathrm{H}_{2} \mathrm{O}$, the elimination of a remaining $\mathrm{H}_{2} \mathrm{O}$, the evolution of gases $\left(4 \mathrm{H}_{2} \mathrm{CO}, 4 \mathrm{CO}\right.$, and $\left.(1 / 2) \mathrm{O}_{2}\right)$, and the generation of copper oxides $\left(\mathrm{CuO}_{2}\right.$ and $\left.\mathrm{CuO}\right)$ take place in the range of 460-584 $\mathrm{K}$. Thus, the theoretical weight loss is calculated to be $50.1 \%(=(18.02+4 \times 30.03+4 \times 28.01+16.00) / 531.33)$. Similarly, the evolution of gases $\left(4 \mathrm{H}_{2} \mathrm{CO}\right.$ and $\left.3 \mathrm{CO}\right)$ and the generation of copper compounds $\left(\mathrm{CuO}_{2}\right.$ and $\left.\mathrm{CuCO}_{3}\right)$ also take place in the range of 460-546 $\mathrm{K}$ for DL- $\mathrm{CuC}_{4} \mathrm{H}_{4} \mathrm{O}_{6} \cdot 2 \mathrm{H}_{2} \mathrm{O}$, and the theoretical weight loss is calculated to be $41.2 \%(=(4 \times 30.03+3 \times 28.01) / 495.30)$. With increased temperature, the theoretical weight losses in the temperature ranges of 584-640 and 640-1250 $\mathrm{K}$ for $\mathrm{L}-\mathrm{CuC}_{4} \mathrm{H}_{4} \mathrm{O}_{6} \cdot 3 \mathrm{H}_{2} \mathrm{O}$ are calculated to be $3.0 \%(=16.00 / 531.33)$ because of the evolution of $(1 / 2) \mathrm{O}_{2}$ gases, which is represented in the third and fourth equations corresponding to the ranges. According to the equations for DL- $\mathrm{CuC}_{4} \mathrm{H}_{4} \mathrm{O}_{6} \cdot 2 \mathrm{H}_{2} \mathrm{O}$ in the temperature ranges of 546-640 K and $640-1250 \mathrm{~K}$, the theoretical weight losses due to the evolutions of $\mathrm{CO}$ and $(1 / 2) \mathrm{O}_{2}$ gases, and of $\mathrm{O}_{2}$ gas are calculated to be $8.9 \%(=(28.01+16.00) / 495.30)$ and $6.5 \%$ $(=32.00 / 495.30)$, respectively. Weight losses based on the chemical reactions for $\mathrm{L}-\mathrm{CuC}_{4} \mathrm{H}_{4} \mathrm{O}_{6} \cdot 3 \mathrm{H}_{2} \mathrm{O}$ and DL- $\mathrm{CuC}_{4} \mathrm{H}_{4} \mathrm{O}_{6} \cdot 2 \mathrm{H}_{2} \mathrm{O}$ are listed in Table 5, and are almost close to the experimental loss values at each temperature range, respectively.

(a)

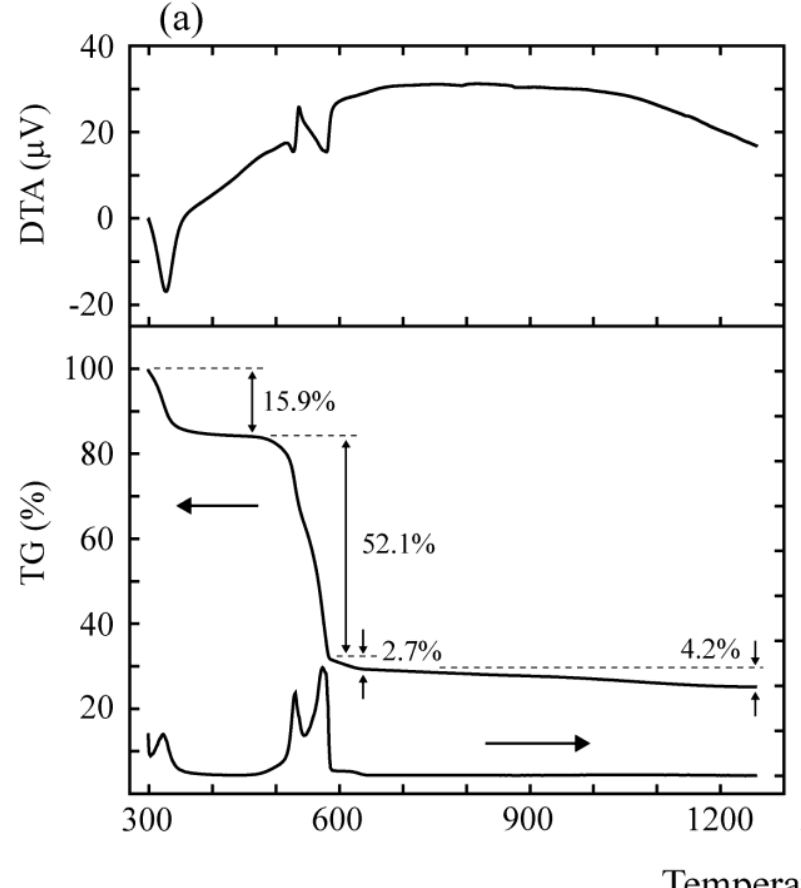

(b)

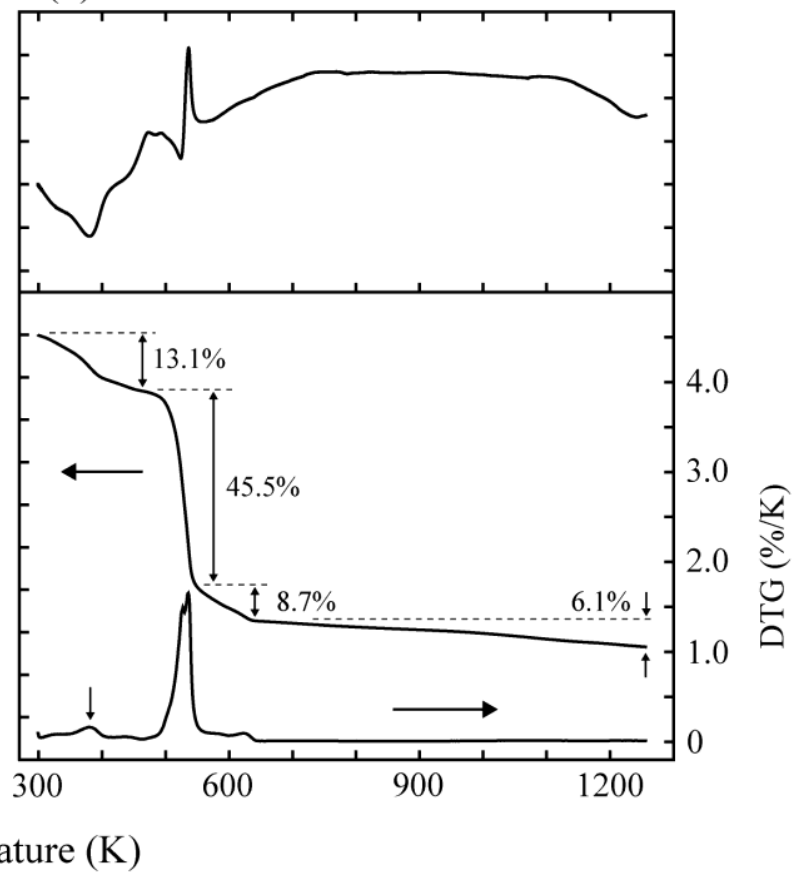

Figure 3. TG, DTG, and DTA curves for (a) $\mathrm{L}-\mathrm{CuC}_{4} \mathrm{H}_{4} \mathrm{O}_{6} \cdot 3 \mathrm{H}_{2} \mathrm{O}$ and (b) DL- $\mathrm{CuC}_{4} \mathrm{H}_{4} \mathrm{O}_{6} \cdot 2 \mathrm{H}_{2} \mathrm{O}$ crystals during heating

The total theoretical weight losses of the $\mathrm{L}-\mathrm{CuC}_{4} \mathrm{H}_{4} \mathrm{O}_{6} \cdot 3 \mathrm{H}_{2} \mathrm{O}$ and DL- $\mathrm{CuC}_{4} \mathrm{H}_{4} \mathrm{O}_{6} \cdot 2 \mathrm{H}_{2} \mathrm{O}$ crystals are found to be $73.1 \%$ $(=17.0+50.1+3.0+3.0)$ and $71.1 \%(=14.5+41.2+8.9+6.5)$, respectively. These values are almost close to the total experimental weight losses in the range of $300-1250 \mathrm{~K}: 74.9 \%(=15.9+52.1+2.7+4.2)$ and $73.4 \%(=13.1+45.5+8.7+6.1)$, respectively, as given in Table 5 and Fig. 3. After heating up to $1250 \mathrm{~K}$ for the TG-DTA measurements of both crystals, we found that reddish-brown materials were present in the respective vessel. These residual materials are presumed to be copper(I) oxide $\mathrm{Cu}_{2} \mathrm{O}$ based on the chemical reactions highlighted above.

\section{Summary}

Single crystals of $\mathrm{L}-\mathrm{CuC}_{4} \mathrm{H}_{4} \mathrm{O}_{6} \cdot 3 \mathrm{H}_{2} \mathrm{O}$ and DL- $\mathrm{CuC}_{4} \mathrm{H}_{4} \mathrm{O}_{6} \cdot 2 \mathrm{H}_{2} \mathrm{O}$ were grown in silica gel medium using gel technique at room temperature. The structures and thermal properties of these crystals were studied by means of X-ray diffraction, DSC, and TG-DTA. The crystal structures of $\mathrm{L}-\mathrm{CuC}_{4} \mathrm{H}_{4} \mathrm{O}_{6} \cdot 3 \mathrm{H}_{2} \mathrm{O}$ at room temperature and DL-CuC $\mathrm{H}_{4} \mathrm{H}_{4} \cdot 2 \mathrm{H}_{2} \mathrm{O}$ at 114 $\mathrm{K}$ were determined to be monoclinic with space groups $P 2_{1}$ and $P 2_{1} / c$, respectively. The structures consisted of slightly distorted $\mathrm{CuO}_{6}$ octahedra, $\mathrm{C}_{4} \mathrm{H}_{4} \mathrm{O}_{6}$ and $\mathrm{H}_{2} \mathrm{O}$ molecules, $\mathrm{C}_{4} \mathrm{H}_{4} \mathrm{O}_{6}-\mathrm{Cu}-\mathrm{C}_{4} \mathrm{H}_{4} \mathrm{O}_{6}$ chains linked by $\mathrm{Cu}-\mathrm{O}$ bonds, and $\mathrm{O}-\mathrm{H}-\mathrm{O}$ hydrogen-bonding frameworks between adjacent molecules. In both crystals, no phase transition was observed in the temperature range of 100-310 K, and the weight losses due to thermal decomposition were found to occur in the temperature range of 300-1250 K. The chemical equations illustrating the decomposition reaction of the crystals were presented, with corresponding temperature ranges. We suggested that the weight losses are caused by the evaporation of 
bound $\mathrm{H}_{2} \mathrm{O}$ molecules, and the evolution of $\mathrm{H}_{2} \mathrm{CO}, \mathrm{CO}$, and $\mathrm{O}_{2}$ gases from $\mathrm{C}_{4} \mathrm{H}_{4} \mathrm{O}_{6}$ molecules. The residual reddish-brown substances left in the vessel after decomposition were identified as copper(I) oxide $\mathrm{Cu}_{2} \mathrm{O}$.

\section{References}

Abdel-Kader, M. M., El-Kabbany, F., Taha, S., Abosehly, A. M., Tahoon, K. K., \& El-Sharkawy, A. A. (1991). Thermal and electrical properties of ammonium tartrate. J. Phys. Chem. Solids, 52(5), 655-658. https://doi.org/10.1016/0022-3697(91)90163-T

Binitha, M. P., \& Pradyumnan P. P. (2013). Thermal degradation, dielectric and magnetic studies on copper tartrate trihydrate crystals. J. Therm. Anal. Calorim., 114(2), 665-669. https://doi.org/10.1007/s10973-013-2998-2

Bootsma, G. A., \& Schoone, J. C. (1967). Crystal structures of mesotartaric acid. Acta Crystallogr., 22(4), 522-532. https://doi.org/10.1107/S0365110X67001070

Bridle, C., \& Lomer, T. R. (1965). The growth of crystals in silica gel, and the unit-cell dimensions of cadmium oxalate and copper tartrate. Acta Crystallogr., 19, 483-484. https://doi.org/10.1107/S0365110X65003699

Burla, M. C., Caliandro, R., Carrozzini, B., Cascarano, G. L., Cuocci, C., Giacovazzo, C., ... Polidori, G. (2015). Crystal structure determination and refinement via SIR2014. J. Appl. Crystallogr., 48(1), 306-309. https://doi.org/10.1107/S1600576715001132

Desai, C. C., \& Patel, A. H. (1988). Crystal data for ferroelectric $\mathrm{RbHC}_{4} \mathrm{H}_{4} \mathrm{O}_{6}$ and $\mathrm{NH}_{4} \mathrm{HC}_{4} \mathrm{H}_{4} \mathrm{O}_{6}$ crystals. J. Mater. Sci. Lett., 7(4), 371-373. https://doi.org/10.1007/BF01730747

Farrugia, L. J. (2012). WinGX and ORTEP for Windows: an update. J. Appl. Crystallogr., 45(4), 849-854. https://doi.org/10.1107/S0021889812029111

Firdous, A., Quasim, I., Ahmad, M. M., \& Kotru, P. N. (2010). Dielectric and thermal studies on gel grown strontium tartrate pentahydrate crystals. Bull. Mater. Sci., 33(4), 377-382. https://doi.org/10.1007/s12034-010-0057-1

Fukami, T., Hiyajyo, S., Tahara, S., \& Yasuda, C. (2017). Thermal properties and crystal structure of $\mathrm{BaC}_{4} \mathrm{H}_{4} \mathrm{O}_{6}$ single crystals, Inter. J. Chem., 9(1), 30-37. https://doi.org/10.5539/ijc.v9n1p30

Fukami, T., Tahara, S., Yasuda, C., \& Nakasone, K. (2016). Structural refinements and thermal properties of $\mathrm{L}(+)$-tartaric, $\mathrm{D}(-)$-tartaric, and monohydrate racemic tartaric acid. Inter. J. Chem., 8(2), 9-21. https://doi.org/10.5539/ijc.v8n2p9

Fukami, T., \& Tahara, S. (2018). Structural and thermal studies on racemic $\mathrm{PbC}_{4} \mathrm{H}_{4} \mathrm{O}_{6} \cdot 2 \mathrm{H}_{2} \mathrm{O}$ single crystal. Chem. Sci. Int. J., 25(1), 45004(1-9). https://doi.org/10.9734/CSJI/2018/45004

Fukami, T., \& Tahara, S. (2020). Crystal structures and thermal properties of $\mathrm{L}-\mathrm{MnC}_{4} \mathrm{H}_{4} \mathrm{O}_{6} \cdot 2 \mathrm{H}_{2} \mathrm{O}$ and DL- $\mathrm{MnC}_{4} \mathrm{H}_{4} \mathrm{O}_{6} \cdot 2 \mathrm{H}_{2} \mathrm{O}$, Inter. J. Chem., 12(1), 78-88. https://doi.org/10.5539/ijc.v12n1p78

Jethva, H. O., Dabhi, R. M., \& Joshi, M. J. (2016). Structural, spectroscopic, magnetic and thermal studies of gel-grown copper levo-tartrate and copper dextro-tartrate crystals. IOSR J. Applied Phys., 8(3), 33-42. https://doi.org/10.9790/4861-0803033342

Labutina, M. L., Marychev, M. O., Portnov, V. N., Somov, N. V., \& Chuprunov, E. V. (2011). Second-order nonlinear susceptibilities of the crystals of some metal tartrates. Crystallogr. Rep., 56(1), 72-74. https://doi.org/10.1134/S1063774510061082

Sheldrick, G. M. (2015). Crystal structure refinement with SHELXL. Acta Crystallogr., C71(1), 3-8. https://doi.org/10.1107/S2053229614024218

Song, Q. B., Teng, M. Y., Dong, Y., Ma, C. A., \& Sun, J. (2006). (2S,3S)-2,3-Dihydroxy-succinic acid monohydrate. Acta Crystallogr., E62(8), o3378-03379. https://doi.org/10.1107/S1600536806021738

Soylu, H. (1996). The structure and molecular structure of copper L-tartrate, $\mathrm{CuC}_{4} \mathrm{H}_{4} \mathrm{O}_{6} \cdot 3 \mathrm{H}_{2} \mathrm{O}$. Acta Crystallogr., A52, C301. https://journals.iucr.org/a/issues/1996/a1/00/a41452/a41452.pdf

Torres, M. E., Peraza, J., Yanes, A. C., López, T., Stockel, J., López, D. M., Solans, X., Bocanegra, E., \& Silgo, G. G. (2002). Electrical conductivity of doped and undoped calcium tartrate. J. Phys. Chem. Solids, 63(4), 695-698. https://doi.org/10.1016/S0022-3697(01)00216-5

\section{Copyrights}

Copyright for this article is retained by the author(s), with first publication rights granted to the journal.

This is an open-access article distributed under the terms and conditions of the Creative Commons Attribution license (http://creativecommons.org/licenses/by/4.0/). 